\title{
High-field electron spin resonance of spin labels in membranes
}

\author{
Derek Marsh ${ }^{\mathrm{a}, *}$, Dieter Kurad ${ }^{\mathrm{a}}$, Vsevolod A. Livshits ${ }^{\mathrm{b}}$ \\ ${ }^{a}$ Max-Planck-Institut für biophysikalische Chemie, Abteilung Spektroskopie, Göttingen 37070, Germany \\ ${ }^{\mathrm{b}}$ Centre of Photochemistry, Russian Academy of Sciences, Moscow 117427, Russia
}

\begin{abstract}
High-field electron spin resonance (ESR) spectroscopy is currently undergoing rapid development. This considerably increases the versatility of spin labelling which, at conventional field strengths, is already well established as a powerful physical technique in membrane biology. Among the unique advantages offered by high-field spectroscopy, particularly for spin-labelled lipids, are sensitivity to non-axial rotation and lateral ordering, a better orientational selection, an extended application to rotational dynamics, and an enhanced sensitivity to environmental polarity. These areas are treated in some depth, along with a detailed consideration of recent developments in the investigation of transmembrane polarity profiles. (C) 2002 Elsevier Science Ireland Ltd. All rights reserved.
\end{abstract}

Keywords: Spin label; Electron spin resonance; Lipid mobility; Rotational diffusion; Lateral ordering; Membrane polarity

\section{Introduction}

Electron spin resonance (ESR) spectroscopy of spin-labelled lipids has long proved to be a valuable method for studying rotational dynamics and molecular ordering in lipid bilayers and biological membranes (Marsh, 1981, 1989; Marsh and Horváth, 1989). The sensitivity arises from the angular anisotropy of the ESR spectrum with respect to the orientation of the spectrometer magnetic field to the spin label magnetic axes (see Fig. 1). This is coupled with the well-known sensitivity of magnetic resonance spectra to exchange processes,

\footnotetext{
* Corresponding author. Tel.: +49-551-201-1285; fax: + 49-551-201-1501.

E-mail address: dmarsh@gwdg.de (D. Marsh).
}

in this case by rotational diffusion between different angular orientations. To exhibit visible effects on the ESR spectrum, the rate of rotational motion must be comparable to the angular frequency equivalent of the spectral extent covered by the rotation. As the rotation rate increases, the spectral lines corresponding to the spin-label $x, y$ and $z$-axes first broaden, next begin to move towards one another, then coalesce to an intermediate average position, and finally narrow about the collapsed position. In the slow motional regime, the linewidths and line shifts are a measure of the rotational rate (Freed, 1976). In the fast motional regime, the residual spectral anisotropy is a measure of the molecular ordering or angular motional amplitude, and the linewidths are determined by the rotational frequency or correlation time (Seelig, 1976). 
In conventional spin-label ESR spectroscopy at an operating frequency of $9 \mathrm{GHz}, g$-value anisotropy makes little contribution. Spectral anisotropy is dominated by the hyperfine interaction with the ${ }^{14} \mathrm{~N}$-nucleus of the nitroxide spin label. The ESR spectrum is then sensitive to rotational correlation times in the range $10^{-10}-10^{-8} \mathrm{~s}$ (see, e.g., Marsh and Horváth, 1989), with optimum sensitivity in the nanosecond regime. This corresponds exactly to the timescale of rotational motion of the lipids in biological membranes. In consequence, spin-label ESR at conventional frequencies and field strengths has become a standard technique for studying lipid dynamics in membranes. This has proved to be particularly fruitful in the case of lipid-protein interactions (see Marsh and Horváth, 1998). Because the rate of rotational motion required for averaging the angular spectral anisotropy matches that of the lipid chain motions in fluid bilayers, perturbations by membrane proteins are readily detected. By contrast, NMR spectroscopy is motionally sensitive down to much lower frequencies; most lipid motions are well into the fast motional regime and NMR spectra are less directly sensitive to lipid-protein interactions.

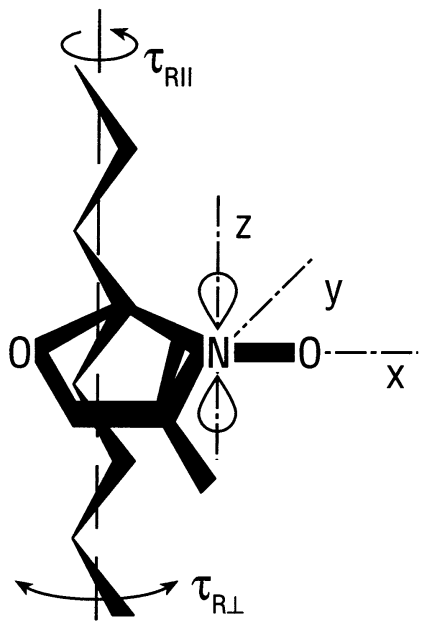

Fig. 1. Magnetic axes of an oxazolidine- $N$-oxyl spin-label group; $x$ is directed along the $\mathrm{N}-\mathrm{O}$ bond and $z$ is perpendicular to the $\mathrm{C}-\mathrm{N}(\mathrm{O})-\mathrm{C}$ plane (i.e. parallel to the axis of the $2 p \pi$-orbital). Attachment of the spin label to a lipid chain (all-trans axis given by the dashed line) is also indicated.
Table 1

Unique features of high-field spin-label ESR spectra

\begin{tabular}{ll}
\hline $\begin{array}{l}\text { Spectral } \\
\text { parameter }\end{array}$ & Molecular property \\
\hline $\begin{array}{l}g_{x x}-g_{y y} \\
\text { anisotropy } \\
\text { Line } \\
\text { broadening/ } \\
\text { shifts }\end{array}$ & Non-axial rotation/ordering \\
$\begin{array}{c}\text { Line narrowing } \\
\text { Fast rotation correlation times: } \\
10^{-10}-10^{-12} \mathrm{~s} \text { and rotational diffusion } \\
\text { anisotropy }\end{array}$ \\
$\begin{array}{c}\text { Environmental polarity } \\
\text { element }\end{array}$ & $\begin{array}{l}\text { Resolution of different } \\
\text { environments/spin-label species }\end{array}$ \\
$g$ - isotropic \\
\hline
\end{tabular}

Whereas the membrane applications of spin-label ESR at normal fields and operating frequencies are well established (see Berliner and Reuben, 1989; Berliner, 1998), those at high fields are still comparatively in their infancy. Due to the considerable potential of the latter, and, because of the recent commercial availability of high-field spectrometers, the present review will concentrate mostly on this area. Methods and applications at conventional fields are adequately reviewed in the references already given.

The unique advantage of high-field spectroscopy over normal spin-label methods is that the $g$-value anisotropy is greater than that of the ${ }^{14} \mathrm{~N}$-hyperfine splittings. Only then are features corresponding to the spin label $x$-, $y$ - and $z$-orientations completely resolved in the powder spectra from non-aligned membrane dispersions. This orientational site selection allows detailed examination of rotational motions about all three spin label axes. Some of the principal features of highfield spectroscopy that are to be discussed here are listed in Table 1. Especially prominent among these are the detection of non-axial (i.e. $x-y$ ) ordering and use of the $g_{x x}$-element as a sensitive indicator of environmental polarity. A catalogue of $94 \mathrm{GHz}$ spectra from spin labels in different environments is given by Smirnov et al. (1998). This also gives some idea of the breadth of possible applications. 


\section{Practical considerations}

From a pragmatic point of view, high-field spectroscopy for nitroxide spin labels can be defined by conditions under which the canonical $x-, y$ - and $z$-orientational features are fully resolved in powder spectra. Practically this corresponds to microwave operating frequencies of 94 $\mathrm{GHz}$ (the so-called W-band) or higher. This is $10 \times$ the normal operating frequency of 9-9.5 $\mathrm{GHz}$, and is the highest frequency of a commercially available spectrometer (from Bruker Analytik). A superconducting magnet with field of 3.5 $\mathrm{T}$ at $94 \mathrm{GHz}$ is required, but adequate sweep widths $(30-60 \mathrm{mT})$ can be provided by room temperature coils.

A high-Q $\mathrm{TE}_{011}$-mode cylindrical cavity resonator can be employed for microwave frequencies up to $140 \mathrm{GHz}$. Beyond this, relatively less sensitive Fabry Perot-type etalons and quasi-optical techniques are required. Sample handling is much more tricky than at the normal $9 \mathrm{GHz}$ (X-band) microwave frequency, especially for non-frozen aqueous samples. Quartz sample capillaries with ID of $0.2 \mathrm{~mm}$ at $94 \mathrm{GHz}$ and $0.1 \mathrm{~mm}$ at $140 \mathrm{GHz}$ are advisable for cylindrical cavity resonators. As always with ESR of membrane samples, pelleting and removal of excess supernatant helps to reduce dielectric loss. This is particularly critical at high microwave frequencies, as is the exact positioning of the sample capillary in the resonator. Addition of glycerol can also serve to reduce dielectric loss of aqueous media. For etalon systems, quite different sample configurations are required, frequently as a thin film on the surface of one of the mirrors.

For general high-field spin-label applications with membranes, a W-band (i.e. $94 \mathrm{GHz}$ ) spectrometer is possibly most versatile. As will be seen later, the W-band spectra still retain the sensitivity to molecular mobility that accounts for much of the success of normal spin-label methodology. At much higher fields, such motions may be driven into the conventional slow motion regime or even to a pseudo-rigid limit, if the increased Zeeman anisotropy is considerably larger than the rotational frequency.

\section{Orientational selection and $g$-value anisotropy}

The basic philosophy behind high-field spectroscopy can be seen from the spin Hamiltonian for a nitroxide free radical:

$$
\mathscr{H}_{s}=\beta_{\mathrm{e}} \mathbf{H} \cdot \mathbf{g} \cdot \mathbf{S}+\mathbf{I} \cdot \mathbf{A} \cdot \mathbf{S}
$$

Here, the first term represents the interaction of the unpaired electron spin $\mathbf{S}$ with the magnetic field $\mathbf{H}$, where $\beta_{\mathrm{e}}$ is the Bohr magneton and $\mathbf{g} \equiv\left(g_{x x}, g_{y y}, g_{z z}\right)$ is the $g$-value tensor. This term specifies the spectral resonance position. The second term is the interaction with the ${ }^{14} \mathrm{~N}$ nuclear spin, I, which gives rise to field-independent hyperfine splittings, where $\mathbf{A} \equiv\left(A_{x x}, A_{y y}, A_{z z}\right)$ is the hyperfine tensor, which has nearly axial symmetry, i.e., $A_{x x} \approx A_{y y}$.

In the high-field approximation, the dependence of the nitroxide spin Hamiltonian on the orientation of the magnetic field, relative to the spin label axes is given by:

$$
\begin{aligned}
& \mathscr{H}_{\mathrm{s}}(\theta, \phi) \\
& =\left[g_{\mathrm{o}}+\frac{1}{3}\left(3 \cos ^{2} \theta-1\right) \Delta g+\sin ^{2} \theta \cos 2 \phi \delta g\right] \\
& \quad \times \beta_{\mathrm{e}} H S_{Z}+\left[a_{\mathrm{o}}+\frac{1}{3}\left(3 \cos ^{2} \theta-1\right) \Delta A\right] I_{Z} S_{Z}
\end{aligned}
$$

where $(\theta, \phi)$ are the polar angles of the magnetic field direction, $Z$, in the nitroxide $x, y, z$-axis system (see Fig. 1). The isotropic $\left(g_{\mathrm{o}}\right)$, and axial $(\Delta g)$ and non-axial $(\delta g)$ anisotropic components of the $g$-tensor are given, respectively, by:

$g_{\mathrm{o}}=\frac{1}{3}\left(g_{x x}+g_{y y}+g_{z z}\right)$

$\Delta g=g_{z z}-\frac{1}{2}\left(g_{x x}+g_{y y}\right)$

$\delta g=\frac{1}{2}\left(g_{x x}-g_{y y}\right)$

Similar expressions hold for the hyperfine tensor. These spin Hamiltonian tensor elements (deduced from high-field, rigid-limit powder spectra) are given for three DOXYL lipid spin labels in Table 2. The significance of the Zeeman anisotropies $\Delta g \beta_{\mathrm{e}} H$ and $\delta g \beta_{\mathrm{e}} H$, relative to that of 
the hyperfine splitting $\Delta A$ can be deduced from these values. First at an ESR frequency of 35 $\mathrm{GHz}(H \sim 1.3 \mathrm{~T})$ does the axial anisotropy of the $g$-tensor $\Delta g \beta_{\mathrm{e}} H$ become comparable to that of the hyperfine splitting $\Delta A$. Only at $94 \mathrm{GHz}(H \sim 3.5$ $\mathrm{T})$, however, does the non-axial $g$-tensor anisotropy $\delta g \beta_{\mathrm{e}} H$ become comparable to the axial hyperfine anisotropy $\Delta A$.

The improvement in orientational resolution with increasing field strength is illustrated for quasi-powder spectra in Fig. 2. This gives the ESR spectra of a chain-labelled lipid spin label in random dispersions of highly ordered bilayer membranes. Spectra are displayed at different field strengths/resonance frequencies. At $9 \mathrm{GHz}$, the only feature unambiguously identified is the $A_{z z}$ outer hyperfine splitting; $x$ - and $y$-features remain essentially unresolved in the centre of the spectrum. At $35 \mathrm{GHz}$, both the $A_{z z}$ hyperfine splitting and the $g_{z z}$-orientational feature are resolved, but the low-field hyperfine line from this manifold and all $x$ - and $y$-spectral features are crowded together at low field. Only at $94 \mathrm{GHz}$, are all three $g_{x x}, g_{y y}$ and $g_{z z}$ features fully resolved, resulting in a complete orientational selection. In addition, all three ${ }^{14} \mathrm{~N}$-hyperfine lines are resolved in the $z$ - and $y$-regions. This is not the case for the low-field $x$-region, because of additional inhomogeneous broadening associated with environmental polarity effects that will be discussed later.

The spin Hamiltonian given by Eq. (2) also allows one to estimate the correlation times at which rotational averaging of the $g_{x x}, g_{y y}$ and $g_{z z}$ spectral features begins. For this to take place, the rotational rate must be greater than the angular spectral anisotropy to be averaged. Taking the nitroxide $z$-axis (see Fig. 1) as the principal axis about which rotation takes place, the critical correlation times for axial and off-axial rotation are, respectively:

$$
\begin{gathered}
\tau_{\text {axial }} \leq \frac{\hbar}{\beta_{\mathrm{e}} H|\delta g|} \\
\tau_{\text {off-axis }} \leq \frac{\hbar}{\beta_{\mathrm{e}} H|\Delta g|}
\end{gathered}
$$

where $\hbar$ is Planck's constant divided by $2 \pi$. In Fig. $1, \tau_{\mathrm{R} \|}$ corresponds to $\tau_{\text {axial }}$ and $\tau_{\mathrm{R} \perp}$ corre-

Table 2

Magnetic tensor elements for 3-DOXYL-17 $\beta$-hydroxy-5 $\alpha$-androstane (ASL; (Smirnova et al., 1995)), 3-DOXYL-cholestane (CSL; (Earle et al., 1993)) and 5-DOXYL stearic acid (5-SASL; (Gaffney and Marsh, 1998)) in solid host matrices

\begin{tabular}{lrrr}
\hline & \multicolumn{1}{c}{ ASL $^{\mathrm{a}}$} & $\mathrm{CSL}^{\mathrm{b}}$ & \\
\hline$g_{x x}$ & $2.00913 \pm 3 \times 10^{-5}$ & $2.00855 \pm 3 \times 10^{-5}$ & $5-\mathrm{SASL}^{\mathrm{c}}$ \\
$g_{y y}$ & $2.0061 \pm 3 \times 10^{-5}$ & $2.00574 \pm 3 \times 10^{-5}$ & $2.00895 \pm 5 \times 10^{-5}$ \\
$g_{z z}$ & $2.00231 \pm 3 \times 10^{-5}$ & $2.00221 \pm 3 \times 10^{-5}$ & $2.00257 \pm 5 \times 10^{-5}$ \\
$g_{\mathrm{o}}$ & $2.00585 \pm 5 \times 10^{-5}$ & $2.00550 \pm 5 \times 10^{-5}$ & $2.00590 \pm 5 \times 10^{-5}$ \\
$\Delta g$ & $-0.00530 \pm 6 \times 10^{-5}$ & $-0.00494 \pm 6 \times 10^{-5}$ & $-0.0050 \pm 1 \times 10^{-4}$ \\
$\delta g$ & $0.00151 \pm 3 \times 10^{-5}$ & $0.00141 \pm 3 \times 10^{-5}$ & $0.0014 \pm 5 \times 10^{-5}$ \\
$A_{x x}$ & $0.53 \pm 0.02 \mathrm{mT}$ & $0.71 \pm 0.02 \mathrm{mT}$ & - \\
$A_{y y}$ & $0.49 \pm 0.02 \mathrm{mT}$ & $0.45 \pm 0.02 \mathrm{mT}$ & $0.66 \pm 0.02 \mathrm{mT}$ \\
$A_{z z}$ & $3.24 \pm 0.02 \mathrm{mT}$ & $3.02 \pm 0.04 \mathrm{mT}$ & $3.37 \pm 0.02 \mathrm{mT}$ \\
$a_{\mathrm{o}}$ & $1.42 \pm 0.03 \mathrm{mT}$ & $1.39 \pm 0.05 \mathrm{mT}$ & $1.56 \pm 0.04 \mathrm{mT}$ \\
$\Delta A$ & $2.73 \pm 0.04 \mathrm{mT}$ & $2.44 \pm 0.06 \mathrm{mT}$ & $2.71 \pm 0.04 \mathrm{mT}$ \\
$\delta A$ & $0.02 \pm 0.02 \mathrm{mT}$ & $0.13 \pm 0.02 \mathrm{mT}$ & - \\
\hline
\end{tabular}

\footnotetext{
${ }^{14} \mathrm{~N}$-hyperfine tensor and $g$-tensor elements are given by $A_{i i}$ and $g_{i i}$, respectively, with $i \equiv x, y, z$ as defined in Fig. $1 . g_{\mathrm{o}}=1 / 3$ $\left(g_{x x}+g_{y y}+g_{z z}\right) ; \Delta g=g_{z z}-1 / 2\left(g_{x x}+g_{y y}\right) ; \delta g=1 / 2\left(g_{x x}-g_{y y}\right)$ and similarly for $a_{\mathrm{o}}, \Delta A$ and $\delta A$.

a 3-DOXYL-17 $\beta$-hydroxy-5 $\alpha$-androstane in $o$-xylene at $-135{ }^{\circ} \mathrm{C}$ (Smirnova et al. 1995).

b 3-DOXYL-cholestane in toluene at $-123{ }^{\circ} \mathrm{C}$ (Earle et al., 1993).

c 5-DOXYL-stearic acid in a dry dimyristoyl phosphatidylcholine:myristic acid 1:2 mol/mol mixture at room temperature (Gaffney and Marsh, 1998).
} 


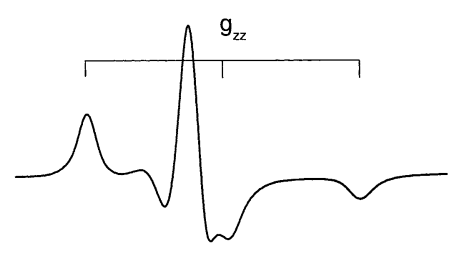

$9 \mathrm{GHz}$

\begin{tabular}{llllllll}
\hline 324 & 326 & 328 & 330 & 332 & 334 & 336 & 338
\end{tabular}
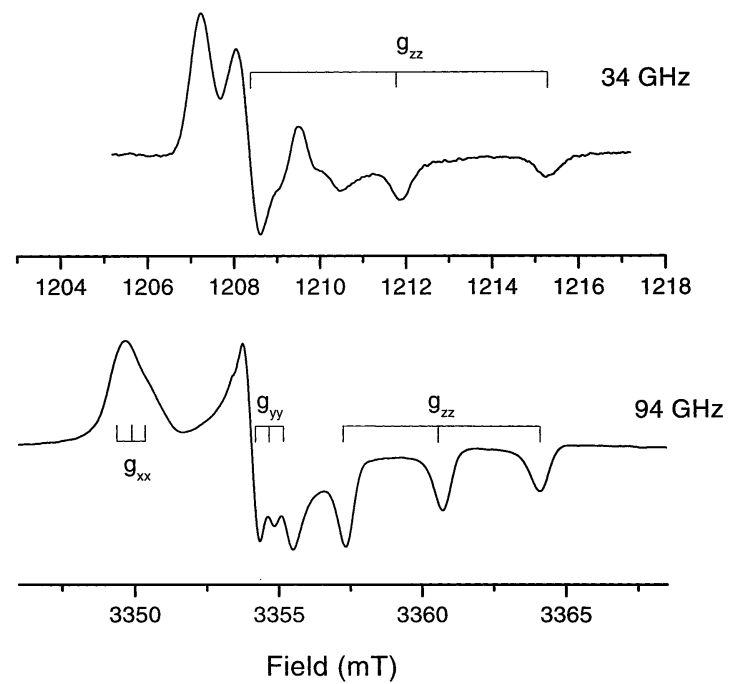

Fig. 2. Multifrequency ESR of a DOXYL spin-labelled lipid in membranes in a quasi-rigid state. ESR spectra of $s n-2$ (5DOXYL) phosphatidylcholine in membranes of dimyristoyl phosphatidylcholine containing $40 \mathrm{~mol} \%$ cholesterol at $10{ }^{\circ} \mathrm{C}$. Spectra are recorded at microwave frequencies of: $9 \mathrm{GHz}$ $(H \sim 0.33 \mathrm{~T}), 34 \mathrm{GHz}(H \sim 1.2 \mathrm{~T})$ and $94 \mathrm{GHz}(H \sim 3.35 \mathrm{~T})$. The width of the magnetic field displays differs for the three spectra shown (Kurad et al., 2001).

sponds to $\tau_{\text {off-axis. }}$ The critical values of these correlation times are listed in Table 3 for different microwave operating frequencies. Clearly, increasingly rapid rotation is required to produce motional averaging as the microwave frequency is increased. For comparison, the critical correlation time for off-axis rotational averaging of the anisotropy in hyperfine splitting is: $\tau_{\text {off-axis }} \leq \hbar /$ $\left(g \beta_{\mathrm{e}} \Delta A\right) \sim 2.1 \times 10^{-9} \mathrm{~s}$, independent of the magnetic field strength. The sensitivity of the spectrum to axial rotation about the $z$-axis, which is exhibited only by the $g$-value anisotropy, therefore first becomes comparable to the sensitivity to off-axial rotation in high-field ESR at $94 \mathrm{GHz}$.

\section{Slow motion with orientational selection}

The slow motional regime corresponds to ESR spectra that are not motionally averaged and still retain the orientational selection characteristic of rigid-limit powder spectra. Slow rotational diffusion has already been studied at $9 \mathrm{GHz}$ microwave frequencies by means of the spectral broadening and inward shifts of the outer $z$-axis extrema, relative to the rigid limit spectrum (Freed, 1976). The improved orientational resolution at high microwave operating frequencies allows such studies at all three, $x, y$ and $z$ canonical positions in the high-field spectra. Complex slow anisotropic rotations and the diffusion mechanism can then be studied in detail. Lebedev (1992) has shown that the relative magnitudes of the shifts and broadening may be used in a diagnostic fashion to determine the mode of rotational diffusion-Brownian, strong jump or librational diffusion - from the high-field spectra.

\subsection{Brownian rotational diffusion}

Lee and Ames (1984) have obtained expressions for the broadening and shift of the canonical extrema in the spectra of radicals undergoing slow isotropic Brownian rotation (see also Lee and Tang, 1985). In the intermediate field approximation, with an axially symmetric spin Hamiltonian, the linebroadening, $1 / T_{2, \mid}\left(m_{I}\right)$, of the parallel extrema is given by:

$\frac{1}{T_{2, \|}\left(m_{I}\right)}=2^{1 / 4} \sqrt{\frac{5}{18}} \sqrt{\frac{\left|H_{\perp}\left(m_{I}\right)-H_{\|}\left(m_{I}\right)\right|}{\gamma_{\mathrm{e}} \tau_{\mathrm{R}}}}$

Table 3

Critical rotational correlation times for motional averaging of $g$-value anisotropy by axial $\left(\tau_{\text {axial }}\right)$ and off-axial $\left(\tau_{\text {off-axis }}\right)$ rotation, at different ESR frequencies

\begin{tabular}{lll}
\hline & $\tau_{\text {axial }}(\mathrm{s})$ & $\tau_{\text {off-axis }}(\mathrm{s})$ \\
\hline $9.4 \mathrm{GHz}$ & $<2.3 \times 10^{-8}$ & $<6.6 \times 10^{-9}$ \\
$34 \mathrm{GHz}$ & $<6.5 \times 10^{-9}$ & $<1.8 \times 10^{-9}$ \\
$94 \mathrm{GHz}$ & $<2.3 \times 10^{-9}$ & $<6.6 \times 10^{-10}$ \\
$140 \mathrm{GHz}$ & $<1.6 \times 10^{-9}$ & $<4.4 \times 10^{-10}$ \\
$250 \mathrm{GHz}$ & $<8.8 \times 10^{-10}$ & $<2.5 \times 10^{-10}$ \\
$360 \mathrm{GHz}$ & $<6.1 \times 10^{-10}$ & $<1.7 \times 10^{-10}$ \\
\hline
\end{tabular}


where $\left|H_{\perp}\left(m_{I}\right)-H_{\|}\left(m_{I}\right)\right|$ is the separation of the parallel and perpendicular extrema in the spectrum, $\gamma_{\mathrm{e}}\left(\equiv g \beta_{\mathrm{e}} / \hbar\right)$ is the electron gyromagnetic ratio, and the Lorentzian half-width is expressed in magnetic field units. The shift in the parallel line position, $\left\langle H_{\|}\left(m_{I}\right)\right\rangle$, from the rigid limit position, $H_{z z}^{\mathrm{o}}$, is given correspondingly by:

$$
\left|\left\langle H_{\|}\left(m_{I}\right)\right\rangle-H_{z z}^{\mathrm{o}}\left(m_{I}\right)\right|=\frac{2^{1 / 4}}{\sqrt{5}} \sqrt{\frac{\left|H_{\perp}\left(m_{I}\right)-H_{\|}\left(m_{I}\right)\right|}{\gamma_{\mathrm{e}} \tau_{\mathrm{R}}}}
$$

In both Eqs. (8) and (9), the isotropic rotational diffusion coefficient is $D_{\mathrm{R}}=1 /\left(6 \tau_{\mathrm{R}}\right)$, where $\tau_{\mathrm{R}}$ is the rotational correlation time. The isotropic average line position is given by $H_{c}=(1 / 3)\left(H_{\|}+\right.$ $\left.2 H_{\perp}\right)$, thus the linebroadening and line shift may be written in the more general form:

$$
\begin{aligned}
& \frac{1}{T_{2, i}\left(m_{I}\right)}=2^{1 / 4} \sqrt{\frac{5}{12}} \sqrt{\frac{\left|H_{i}-H_{c}\right|}{\gamma_{\mathrm{e}} \tau_{\mathrm{R}}}} \\
& \left|\left\langle H_{i}\right\rangle-H_{i i}^{\mathrm{o}}\right|=2^{1 / 4} \sqrt{\frac{3}{10}} \sqrt{\frac{\left|H_{i}-H_{c}\right|}{\gamma_{\mathrm{e}} \tau_{\mathrm{R}}}}
\end{aligned}
$$

where the index $i$ represents the $x, y$ or $z$-axis. For slow Brownian rotational diffusion, the linebroadening is comparable in magnitude to the shift in line position. This is unlike the situation for strong jump diffusion.

\subsection{Strong jump diffusion}

The strong jump diffusion model has been parameterised quantitatively in terms of the familiar results for two-site exchange in magnetic resonance (Freed, 1976). The increase in transverse relaxation rate, $1 / T_{2}$, is equal to the two-site jump rate, $1 / \tau_{\mathrm{J}}$. The contribution to the linewidth is therefore simply:

$\frac{1}{T_{2}\left(m_{I}\right)}=\frac{1}{\gamma_{e} \tau_{\mathbf{J}}}$

in magnetic field units. The linewidths are therefore inversely proportional to $\tau$ for strong jump diffusion, as opposed to the weaker $\tau^{-1 / 2}$ dependence for Brownian rotational diffusion. For twosite exchange between sites with resonance fields $H_{A}$ and $H_{B}$, the reduction in spectral splitting is given by (Gutowsky and Holm, 1956):

$$
H_{A}-H_{B}=\left(H_{A}-H_{B}\right)_{\mathrm{o}}\left[1-\frac{8}{\gamma_{\mathrm{e}}^{2}\left(H_{A}-H_{B}\right)_{\mathrm{o}}^{2} \tau_{\mathrm{J}}^{2}}\right]^{1 / 2}
$$

where $\left(H_{A}-H_{B}\right)_{\mathrm{o}}$ is the difference in resonance field in the absence of exchange. In this case, the average line position is $H_{c}=(1 / 2)\left(H_{A}+H_{B}\right)$. For strong jump diffusion, the shift in the canonical resonance position from Eq. (13) is then given by:

$$
\left|\left\langle H_{i}\right\rangle-H_{i i}^{\mathrm{o}}\right|=\frac{1}{\gamma_{\mathrm{e}}^{2}\left|H_{i}-H_{c}\right| \tau_{\mathrm{J}}^{2}}
$$

The shift in line position given by Eq. (13) is a second-order effect and therefore is considerably smaller than that found for Brownian diffusion. From Eq. (14), it can be seen that for strong jump diffusion the shift decreases inversely with microwave frequency and therefore becomes even less significant in high-field spectroscopy.

The relationships between the line broadenings and line shifts for the two different slow rotational diffusion mechanisms and between highfield and normal-field EPR are given in Table 4. Approximate numerical constants are given from the preceding equations, for uniaxial rotation around the $x-, y$-, or $z$-nitroxide principal axes. These values are calculated from the corresponding rigid-limit spin Hamiltonian tensor elements and correspond to the slow-motional limit. These quantitative values allow a qualitative distinction between slow Brownian and strong jump rotational diffusion (Lebedev, 1992), as is indicated in Table 5. For Brownian rotation, line broadenings and shifts are of comparable magnitude, whereas for strong jump diffusion the line shifts are much smaller than the line broadenings. Also included in Table 5 is the effect of fast librational motion of limited amplitude, in an otherwise rigid system. The librations are assumed to be sufficiently fast that they produce no line broadening, but they produce line shifts that are determined by the extent of partial motional averaging of the spectral anisotropy.

An example of this type of analysis applied to $94 \mathrm{GHz}$ spectroscopy in a membrane system is given in Gaffney and Marsh (1998). The off-axis chain flexing motions of spin-labelled phos- 
Table 4

Approximate numerical expressions for the dependence of the linewidths, $1 / T_{2, i}(\mathrm{mT})$, and shifts, $\left\langle\delta H_{i}\right\rangle(m T)$ of the canonical $\mathrm{g}_{i i}$ spectral extrema on the rotational correlation time, $\tau(\mathrm{ns})$, for slow rotational diffusion about the $z-(i \equiv x, y), y-(i \equiv x, z)$ or $x$ $(i \equiv y, z)$ axes

\begin{tabular}{|c|c|c|c|c|c|c|}
\hline \multirow[t]{2}{*}{ AXIS } & \multicolumn{2}{|r|}{$z$} & \multicolumn{2}{|r|}{$y$} & \multicolumn{2}{|r|}{$x$} \\
\hline & $1 / T_{2, x / y}$ & $\left\langle\delta H_{x / y}\right\rangle$ & $1 / T_{2, x / z}$ & $\left\langle\delta H_{x / z}\right\rangle$ & $1 / T_{2, y / z}$ & $\left\langle\delta H_{y / z}\right\rangle$ \\
\hline \multicolumn{7}{|l|}{ Brownian } \\
\hline $360 \mathrm{GHz}$ & $5.45 \tau_{\mathrm{R}}^{-1 / 2}$ & $4.63 \tau_{\mathrm{R}}^{-1 / 2}$ & $8.26 \tau_{\mathrm{R}}^{-1 / 2}$ & $7.01 \tau_{\mathrm{R}}^{-1 / 2}$ & $6.20 \tau_{\mathrm{R}}^{-1 / 2}$ & $5.26 \tau_{\mathrm{R}}^{-1 / 2}$ \\
\hline $250 \mathrm{GHz}$ & $4.54 \tau_{\mathrm{R}}^{-1 / 2}$ & $3.86 \tau_{\mathrm{R}}^{-1 / 2}$ & $6.89 \tau_{\mathrm{R}}^{-1 / 2}$ & $5.84 \tau_{\mathrm{R}}^{-1 / 2}$ & $5.17 \tau_{\mathrm{R}}^{-1 / 2}$ & $4.39 \tau_{\mathrm{R}}^{-1 / 2}$ \\
\hline $140 \mathrm{GHz}$ & $3.40 \tau_{\mathrm{R}}^{-1 / 2}$ & $2.89 \tau_{\mathrm{R}}^{-1 / 2}$ & $5.15 \tau_{\mathrm{R}}^{-1 / 2}$ & $4.37 \tau_{\mathrm{R}}^{-1 / 2}$ & $3.87 \tau_{\mathrm{R}}^{-1 / 2}$ & $3.29 \tau_{\mathrm{R}}^{-1 / 2}$ \\
\hline $94 \mathrm{GHz}$ & $2.79 \tau_{\mathrm{R}}^{-1 / 2}$ & $2.36 \tau_{\mathrm{R}}^{-1 / 2}$ & $4.22 \tau_{\mathrm{R}}^{-1 / 2}$ & $3.58 \tau_{\mathrm{R}}^{-1 / 2}$ & $3.17 \tau_{\mathrm{R}}^{-1 / 2}$ & $2.69 \tau_{\mathrm{R}}^{-1 / 2}$ \\
\hline $34 \mathrm{GHz}$ & $1.68 \tau_{\mathrm{R}}^{-1 / 2}$ & $1.42 \tau_{\mathrm{R}}^{-1 / 2}$ & $2.54 \tau_{\mathrm{R}}^{-1 / 2}$ & $2.16 \tau_{\mathrm{R}}^{-1 / 2}$ & $1.91 \tau_{\mathrm{R}}^{-1 / 2}$ & $1.62 \tau_{\mathrm{R}}^{-1 / 2}$ \\
\hline $9.4 \mathrm{GHz}$ & $0.88 \tau_{\mathrm{R}}^{-1 / 2}$ & $0.75 \tau_{\mathrm{R}}^{-1 / 2}$ & $1.34 \tau_{\mathrm{R}}^{-1 / 2}$ & $1.13 \tau_{\mathrm{R}}^{-1 / 2}$ & $1.00 \tau_{\mathrm{R}}^{-1 / 2}$ & $0.85 \tau_{\mathrm{R}}^{-1 / 2}$ \\
\hline \multicolumn{7}{|c|}{ Strong jump } \\
\hline $360 \mathrm{GHz}$ & $5.68 \tau_{\mathrm{J}}^{-1}$ & $3.63 \tau_{\mathbf{J}}^{-2}$ & $5.68 \tau_{\mathbf{J}}^{-1}$ & $1.58 \tau_{\mathbf{J}}^{-2}$ & $5.68 \tau_{\mathrm{J}}^{-1}$ & $2.80 \tau_{\mathbf{J}}^{-2}$ \\
\hline $250 \mathrm{GHz}$ & $5.68 \tau_{\mathbf{J}}^{-1}$ & $5.23 \tau_{\mathbf{J}}^{-2}$ & $5.68 \tau_{\mathbf{J}}^{-1}$ & $2.27 \tau_{\mathrm{J}}^{-2}$ & $5.68 \tau_{\mathrm{J}}^{-1}$ & $4.03 \tau_{\mathbf{J}}^{-2}$ \\
\hline $140 \mathrm{GHz}$ & $5.68 \tau_{\mathrm{J}}^{-1}$ & $9.33 \tau_{\mathbf{J}}^{-2}$ & $5.68 \tau_{\mathrm{J}}^{-1}$ & $4.06 \tau_{\mathrm{J}}^{-2}$ & $5.68 \tau_{\mathrm{J}}^{-1}$ & $7.19 \tau_{\mathrm{J}}^{-2}$ \\
\hline $94 \mathrm{GHz}$ & $5.68 \tau_{\mathbf{J}}^{-1}$ & $13.90 \tau_{\mathrm{J}}^{-2}$ & $5.68 \tau_{\mathbf{J}}^{-1}$ & $6.05 \tau_{\mathrm{J}}^{-2}$ & $5.68 \tau_{\mathrm{J}}^{-1}$ & $10.71 \tau_{\mathrm{J}}^{-2}$ \\
\hline $34 \mathrm{GHz}$ & $5.68 \tau_{\mathbf{J}}^{-1}$ & $38.4 \tau_{\mathbf{J}}^{-2}$ & $5.68 \tau_{\mathbf{J}}^{-1}$ & $16.7 \tau_{\mathrm{J}}^{-2}$ & $5.68 \tau_{\mathrm{J}}^{-1}$ & $29.6 \tau_{\mathbf{J}}^{-2}$ \\
\hline $9.4 \mathrm{GHz}$ & $5.68 \tau_{\mathrm{J}}^{-1}$ & $139.0 \tau_{\mathrm{J}}^{-2}$ & $5.68 \tau_{\mathrm{J}}^{-1}$ & $60.5 \tau_{\mathrm{J}}^{-2}$ & $5.68 \tau_{\mathrm{J}}^{-1}$ & $107.1 \tau_{\mathrm{J}}^{-2}$ \\
\hline
\end{tabular}

Values are given for both Brownian rotational diffusion $\left(\tau_{\mathrm{R}}\right)$ from Eqs. (10) and (11) and strong jump rotational diffusion $\left(\tau_{\mathrm{J}}\right)$ from Eqs. (12) and (14), and for different operating frequencies. First at $94 \mathrm{GHz}$ are the $g_{x x}$-features $\left(1 / T_{2, x},\left\langle\delta H_{x}\right\rangle\right)$ resolved from the $g_{y y}$-features (see Fig. 2), and rotation about the $z$-axis (in addition to that about the $x$-and $y$-axes) can then be analysed. Below $94 \mathrm{GHz}$, correlation times can be deduced reliably only from the $g_{z z}$-features $\left(1 / T_{2, z},\left\langle\delta H_{z}\right\rangle\right)$ and only rotation about the $x$ - or $y$-axes can be analysed.

phatidylcholine in liquid-ordered membranes containing high cholesterol approximate to Brownian rotational diffusion with effective correlation times in the range $\tau_{\mathrm{R} \perp} \sim 1.5-7.5 \times 10^{-8} \mathrm{~s}$ depending on the position of chain labelling.

\section{Fast anisotropic rotation}

High-field ESR extends the sensitivity of spinlabel ESR to faster rotational correlation times and increases the sensitivity of fast-motion spectra to rotational anisotropy. Although these features have not yet been exploited much in membrane systems, rapid anisotropic segmental motion of spin-labelled cysteine side chains has been detected in an aqueous peptide by ESR at $140 \mathrm{GHz}$ (Bennati et al., 1999). Applications to membraneactive peptides therefore can be anticipated.

In the fast motional regime, the differential line broadening of the three motionally averaged ${ }^{14} \mathrm{~N}$ hyperfine lines is given by:
$\frac{1}{T_{2}\left(m_{I}\right)}=A+B m_{I}+C m_{I}^{2}$

where $m_{I}=-1,0,+1$ is the nuclear magnetic quantum number of the high-, central and lowfield lines, respectively. The linewidth coefficients are related to the rotational correlation times $\tau_{20}$ and $\tau_{22}$ by (see Goldman et al., 1972):

Table 5

Relative values of the shift, $\mid\left\langle H_{i}\right\rangle-H_{i i}^{o}$, and broadening, $\left(1 / T_{2, i}-1 / T_{2, i}^{\mathrm{o}}\right)$, of the canonical extrema for different modes of molecular rotation (see (Lebedev, 1992))

\begin{tabular}{llll}
\hline Shift & Broadening & Mode \\
\hline$\left|\left\langle H_{i}\right\rangle-H_{i i}^{o}\right|$ & $\sim$ & $\left(1 / T_{2, i}-1 / T_{2, i}^{\mathrm{o}}\right)$ & Brownian \\
diffusion \\
$\left|\left\langle H_{i}\right\rangle-H_{i i}^{o}\right|$ & $\ll$ & $\left(1 / T_{2, i}-1 / T_{2, i}^{\mathrm{o}}\right)$ & $\begin{array}{l}\text { Strong jump } \\
\text { diffusion }\end{array}$ \\
$\left|\left\langle H_{i}\right\rangle-H_{i i}^{o}\right|$ & $\gg$ & $\left(1 / T_{2, i}-1 / T_{2, i}^{\mathrm{o}}\right)$ & Fast libration \\
& & $\approx 0$ & \\
\hline
\end{tabular}

Anisotropic rotational diffusion is characterised by differential effects on the $i \equiv x, y$, or $z$ canonical positions. e.g. for preferential rotation around the $z$-axis: $x, y>z$. 
Table 6

Linewidth coefficients in the motional narrowing regime for anisotropic rotation diffusion about the spin-label $z$-, $x$ - and $y$-axes at different ESR frequencies

\begin{tabular}{|c|c|c|c|c|}
\hline Frequency & Coefficients & $z$-axis & $x$-axis & $y$-axis \\
\hline \multirow[t]{3}{*}{$9.4 \mathrm{GHz}$} & $A-A^{\prime}$ & $0.010 \tau_{20}+0.003 \tau_{22}$ & $0.032 \tau_{20}+0.071 \tau_{22}$ & $0.023 \tau_{20}+0.080 \tau_{22}$ \\
\hline & $B$ & $0.076 \tau_{20}+0.0005 \tau_{22}$ & $-0.034 \tau_{20}-0.041 \tau_{22}$ & $-0.003 \tau_{20}-0.073 \tau_{22}$ \\
\hline & $C$ & $0.073 \tau_{20}+0.0001 \tau_{22}$ & $0.017 \tau_{20}+0.056 \tau_{22}$ & $0.019 \tau_{20}+0.054 \tau_{22}$ \\
\hline \multirow[t]{2}{*}{$34 \mathrm{GHz}$} & $A-A^{\prime}$ & $0.249 \tau_{20}+0.039 \tau_{22}$ & $0.160 \tau_{20}+0.128 \tau_{22}$ & $0.024 \tau_{20}+0.264 \tau_{22}$ \\
\hline & $B$ & $-0.274 \tau_{20}+0.002 \tau_{22}$ & $-0.124 \tau_{20}-0.148 \tau_{22}$ & $-0.010 \tau_{20}-0.262 \tau_{22}$ \\
\hline \multirow[t]{2}{*}{$94 \mathrm{GHz}$} & $A-A^{\prime}$ & $1.317 \tau_{20}+0.301 \tau_{22}$ & $1.081 \tau_{20}+0.537 \tau_{22}$ & $0.029 \tau_{20}+1.589 \tau_{22}$ \\
\hline & $B$ & $-0.758 \tau_{20}+0.005 \tau_{22}$ & $-0.344 \tau_{20}-0.409 \tau_{22}$ & $-0.028 \tau_{20}-0.726 \tau_{22}$ \\
\hline \multirow[t]{2}{*}{$140 \mathrm{GHz}$} & $A-A^{\prime}$ & $2.815 \tau_{20}+0.667 \tau_{22}$ & $2.372 \tau_{20}+1.111 \tau_{22}$ & $0.037 \tau_{20}+3.446 \tau_{22}$ \\
\hline & $B$ & $-1.129 \tau_{20}+0.007 \tau_{22}$ & $-0.513 \tau_{20}-0.609 \tau_{22}$ & $-0.041 \tau_{20}-1.081 \tau_{22}$ \\
\hline \multirow[t]{2}{*}{$250 \mathrm{GHz}$} & $A-A^{\prime}$ & $8.785 \tau_{20}+2.128 \tau_{22}$ & $7.517 \tau_{20}+3.396 \tau_{22}$ & $0.068 \tau_{20}+10.845 \tau_{22}$ \\
\hline & $B$ & $-2.016 \tau_{20}+0.013 \tau_{22}$ & $-0.915 \tau_{20}-1.088 \tau_{22}$ & $-0.074 \tau_{20}-1.930 \tau_{22}$ \\
\hline \multirow[t]{2}{*}{$360 \mathrm{GHz}$} & $A-A^{\prime}$ & $18.122 \tau_{20}+4.412 \tau_{22}$ & $15.564 \tau_{20}+6.970 \tau_{22}$ & $0.115 \tau_{20}+22.419 \tau_{22}$ \\
\hline & $B$ & $-2.903 \tau_{20}+0.018 \tau_{22}$ & $-1.318 \tau_{20}-1.567 \tau_{22}$ & $-0.106 \tau_{20}-2.779 \tau_{22}$ \\
\hline
\end{tabular}

Calculated from Eqs. (16)-(18). Correlation times $\tau_{20}$ and $\tau_{22}$ are in ns, and linewidth coefficients $A-A^{\prime}, B$ and $C$ are in mT. The $C$-coefficient is independent of the microwave frequency. Spin Hamiltonian parameters used are those of spin-labelled ASL that are given in Table 2. Values for isotropic rotational diffusion are obtained by putting $\tau_{22}=\tau_{20}\left(\equiv \tau_{\mathrm{R}}\right)$.

$$
\begin{aligned}
A= & A^{\prime}+\frac{4}{45}\left[(\Delta g)^{2} \tau_{20}+3(\delta g)^{2} \tau_{22}\right]\left(\frac{\beta_{\mathrm{e}}}{\hbar}\right)^{2} H^{2} \\
& +\frac{\gamma_{\mathrm{e}}^{2}}{15}\left[(\Delta A)^{2} \tau_{20}+3(\delta A)^{2} \tau_{22}\right] \\
B= & \frac{8}{45} \gamma_{\mathrm{e}}\left(\Delta A \Delta g \tau_{20}+3 \delta A \delta g \tau_{22}\right)\left(\frac{\beta_{\mathrm{e}}}{\hbar}\right) H \\
C= & \frac{\gamma_{\mathrm{e}}^{2}}{18}\left[(\Delta A)^{2} \tau_{20}+3(\delta A)^{2} \tau_{22}\right]
\end{aligned}
$$

where non-secular terms have been neglected and pseudo-secular spectral densities have been put equal to the zero-frequency secular ones. The former assumption is valid for rotational correlation times longer than $10^{-11} \mathrm{~s}$ at $94 \mathrm{GHz}$ and longer than $10^{-12} \mathrm{~s}$ at $250 \mathrm{GHz}$. The latter assumption applies for correlation times shorter than $10^{-9} \mathrm{~s}$, although this value may be decreased at higher fields when the nuclear Zeeman interaction becomes appreciable. Note that the factors of $\gamma_{\mathrm{e}}$ in Eqs. (16)-(18) imply that the hyperfine anisotropies $\Delta A$ and $\delta A$ are expressed in magnetic field units. In Eq. (16), $A^{\prime}$ represents other homogeneous broadening mechanisms such as spin rotation, Heisenberg exchange, etc. This residual line broadening has often precluded use of the $A$-coefficient for determining rotational correlation times from linewidth measurements in normal low-field ESR.
The rotational correlation times, $\tau_{20}$ and $\tau_{22}$, in Eqs. (16)-(18) are defined in terms of second rank spherical tensors. They are related to the principal elements $D_{\mathrm{R} \|}$ and $D_{\mathrm{R} \perp}$ of the axial rotational diffusion tensor by:

$\tau_{20}^{-1}=6 D_{\mathrm{R} \perp}$

and

$\tau_{22}^{-1}=4 D_{\mathrm{R} \|}+2 D_{\mathrm{R} \perp}$

Hence the familiar rotational correlation times $\tau_{\mathrm{R} \|}\left(\equiv 1 / 6 D_{\mathrm{R} \|}\right)$ and $\tau_{\mathrm{R} \perp}\left(\equiv 1 / 6 D_{\mathrm{R} \perp}\right)$ that are depicted in Fig. 1 can be obtained directly from a combination of the different linewidth coefficients. With the tensor anisotropies $\Delta g, \delta g$ and $\Delta A, \delta A$ as defined by Eqs. (3)-(5) and equivalents, the principal rotational axis $\left(R_{\|}\right)$is the nitroxide $z$-axis. Corresponding expressions for axial rotation about the $x$ - and $y$-axes of the nitroxide are obtained by cyclic permutation of the indices in Eqs. (3)-(5). Numerical values for the linewidth coefficients with increasing ESR frequency are presented in Table 6 . These are given in terms of the anisotropic rotational correlation times $\tau_{20}$ and $\tau_{22}$ (cf. Eqs. (19) and (20)), where the nitroxide $z-, x$ - or $y$-axis is the principal rotational axis. 
The advantages of high-field ESR are seen immediately from Table 6 . The quadratic field dependence of the $A$-coefficient means that this term dominates over the residual homogeneous line broadening mechanisms in high-field spectroscopy. In favourable circumstances, inhomogeneous broadening may also be ignored. Further, the $B$-coefficient comes to dominate over the $C$ coefficient at high fields. This is important, because, the latter is relatively insensitive to motional anisotropy, for $z$-axis rotation, because, then the non-axial hyperfine anisotropy $\delta A$ is very small (see Table 2). Finally, the largest linewidth coefficients are much greater at high frequency than at $9 \mathrm{GHz}$. It is this, which confers the sensitivity to faster rotational motions.

Budil et al. (1993) have combined ESR measurements at 250 and $9.5 \mathrm{GHz}$ to determine the $x$-, $y$ - and $z$-elements of the non-axial rotational diffusion tensor of a small spin label (perdeuterated TEMPONE) in a low-viscosity isotropic solvent (deuterated toluene). Combination with 9.5 $\mathrm{GHz}$ measurements was necessary because, fastmotional linewidths at $250 \mathrm{GHz}$ are dominated by the $A$ - and $B$-coefficients and therefore are, relatively insensitive to the $C$-coefficient (see Table 6). This method has been applied to the fast-motional spectra of the spin-labelled steroid androstanol (ASL) in toluene at 94 and $9 \mathrm{GHz}$ (Smirnova et al., 1995). The rotational anisotropy, assumed independent of temperature, is characterised by $\rho_{x}=D_{\mathrm{R}, x} / D_{\mathrm{R}, z}=1.6 \pm 0.5$ and $\rho_{y}=D_{\mathrm{R}, y} / D_{\mathrm{R}, z}=5.8 \pm 1.0$. For this particular spin-labelled lipid (and also the cholestane derivative CSL), the nitroxide $y$-axis is directed approximately along the long molecular axis of the steroid nucleus.

As already mentioned, fast-motional analysis has been applied to study side-chain motion in a helix-forming alanine-based peptide in aqueous solution (Bennati et al., 1999). The peptide was spin-labelled with a methanethiosulphonate derivative (MTSSL) at the single cysteine residue in the peptide. The $140 \mathrm{GHz}$ ESR spectra indicated preferentially rapid rotation about the nitroxide $x$-axis (cf. Table 6) of the spin-labelled side chain with correlation times of $\tau_{\mathrm{R} \|}=0.08 \mathrm{~ns}$ and $\tau_{\mathrm{R} \perp}=0.21 \mathrm{~ns}$ at $33{ }^{\circ} \mathrm{C}$.

\section{Lateral lipid ordering by cholesterol}

Routinely, the chain segments in fatty acids or phospholipids are spin-labelled with the 4,4dimethyl-oxazolidine- $N$-oxyl (DOXYL) moiety. The DOXYL ring (see Fig. 1) is stereospecifically attached with the principal $z$-axis of the ${ }^{14} \mathrm{~N}$-hyperfine tensor parallel to the long axis of the lipid chain. Therefore, ESR spectra at conventional fields are optimally sensitive to orientational order of the lipid chains relative to the membrane normal. This sensitivity to chain ordering and segmental rotational isomerism is the basis of many conventional membrane ESR studies with spin-labelled lipids and accounts for much of the success of the method (see e.g. Marsh, 1981, 1989).

The 9-GHz ESR spectra of conventional DOXYL spin-labelled chains, whilst being optimally sensitive to order of the long axis, are, however, relatively insensitive to lateral chain ordering within the membrane plane. This is, because, the ${ }^{14} \mathrm{~N}$-hyperfine tensor (see Table 2) has close to axial symmetry about the nitroxide $z$ axis, and at conventional magnetic fields $g$-value anisotropy is relatively unimportant. As pointed out in a previous section, to obtain sensitivity of the DOXYL labels to rotation about the chain long axis (i.e. about the nitroxide $z$-axis) it is necessary to use high-field spectroscopy. Recently, we have found that the $g_{x x}$ and $g_{y y}$ features are resolved in the $94 \mathrm{GHz}$ ESR spectra of DOXYL spin-labelled lipid chains when these are incorporated in phospholipid bilayers containing a high concentration of cholesterol (Gaffney and Marsh, 1998; and see e.g. Fig. 2). This non-axiality has allowed study of lateral phospholipid ordering and possible domain formation by cholesterol in liquid-ordered membrane phases (Kurad et al., 2001).

As an example, 94-GHz ESR spectra of bilayer membranes prepared from dimyristoyl phosphatidylcholine with $40 \mathrm{~mol} \%$ cholesterol are given in Fig. 3. Spectra correspond to the liquidordered phase and are given for DOXYL spin labels attached at different $\mathrm{C}$-atom positions in the $s n-2$ chain of phosphatidylcholine. The non- 
axial anisotropy between the $g_{x x}$ - and $g_{y y}$-positions is seen very clearly in the spectra for spin labels down to C-atom $n=10$ in the chain. For 12-PCSL (i.e., $n=12$ ), the $g_{x x}-g_{y y}$ anisotropy is partially collapsed and is completely averaged for 14-PCSL which is spin-labelled close to the termi- nal methyl of the sn-2 chain. For the latter, a single broad first-derivative-like feature is observed at the average $g_{x x}, g_{y y}$-position.

The rotational averaging of the line positions in Fig. 3 can be analysed quantitatively by using the high-field spin Hamiltonian given in Eq. (2). For
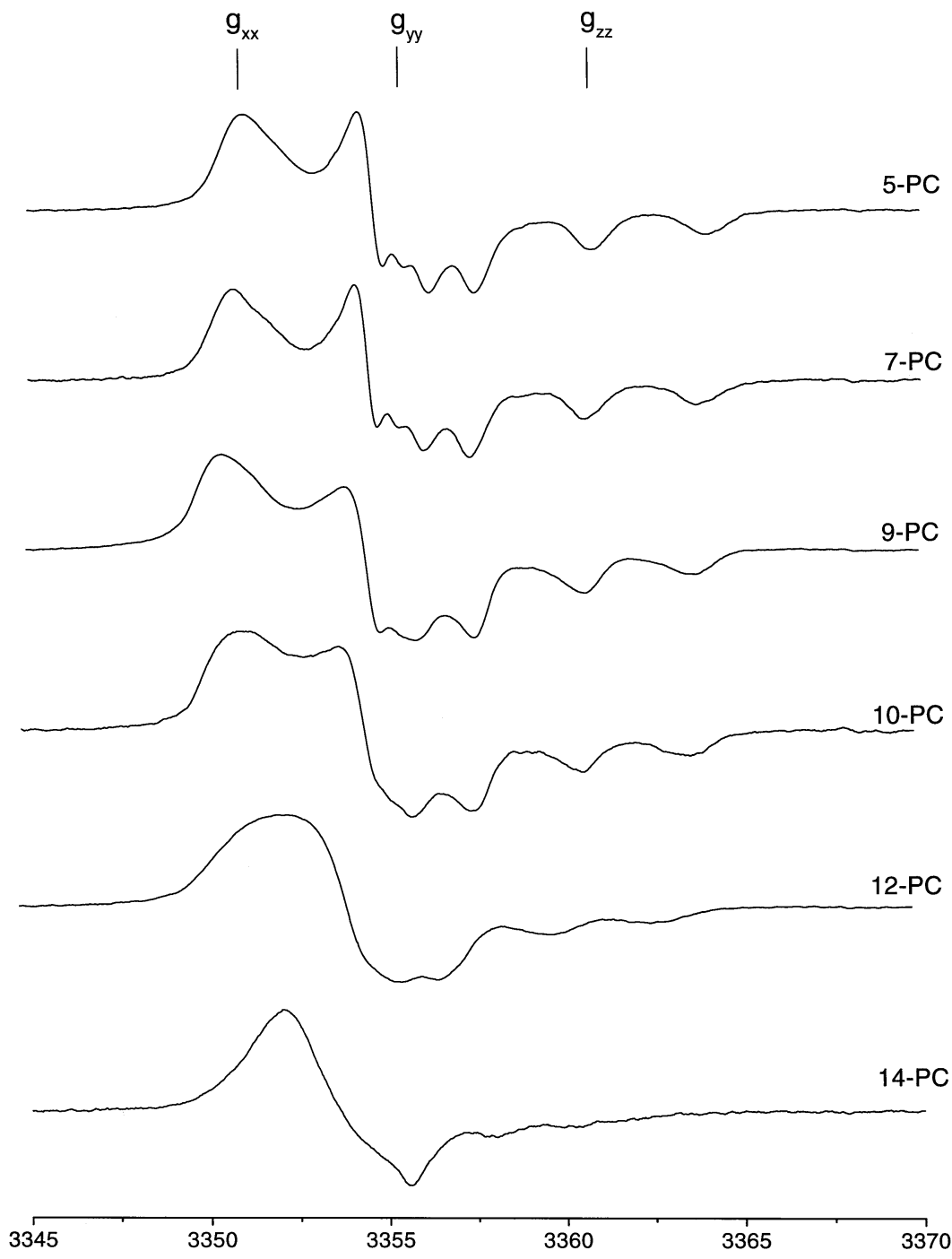

Field (mT)

Fig. 3. High-field 94-GHz ESR spectra of DOXYL-spin-labelled phosphatidylcholine at 0.5 mol\% in fully hydrated membranes of dimyristoyl phosphatidylcholine plus $40 \mathrm{~mol} \%$ cholesterol at $30{ }^{\circ} \mathrm{C}$. The position, $n$, of the spin label in the $s n-2 \mathrm{chain}$ is indicated in the figure (Kurad et al., 2001). 
each ${ }^{14} \mathrm{~N}$-hyperfine manifold, $m_{I}$, the resonance field is given by:

$$
\begin{aligned}
\left\langle H_{\mathrm{res}}\left(m_{I}\right)\right\rangle= & H_{\mathrm{o}}^{\mathrm{res}}\left(m_{I}\right) \\
& -\frac{h v}{g_{\mathrm{o}} \beta_{\mathrm{e}}}\left[\frac{1}{3}\left(\frac{\Delta g}{g_{\mathrm{o}}}+\frac{\Delta A}{h v} m_{I}\right)\right. \\
& \times\left\langle 3 \cos ^{2} \theta-1\right\rangle \\
& \left.+\frac{\delta g}{g_{\mathrm{o}}}\left\langle\sin ^{2} \theta \cos 2 \phi\right\rangle\right]
\end{aligned}
$$

where $H_{\mathrm{o}}^{\mathrm{res}}$ is the isotropic resonance position and $v$ is the microwave frequency. The angular parentheses represent motional averaging over the spin label orientation relative to the static field direction. This rotational averaging is of limited amplitude but is assumed to be fast relative to the spectral anisotropy being averaged. Site selection is retained in the partially averaged powder spectrum, which is characterised by the following effective motionally averaged elements of the $g$-tensor:

$$
\begin{aligned}
& \left\langle g_{z z}\right\rangle \approx g_{\mathrm{o}}+\frac{1}{3} \Delta g\left\langle 3 \cos ^{2} \theta-1\right\rangle \\
& \left\langle g_{x x}\right\rangle \approx g_{\mathrm{o}}-\frac{1}{3} \Delta g+\delta g\langle\cos 2 \phi\rangle \\
& \left\langle g_{y y}\right\rangle \approx g_{\mathrm{o}}-\frac{1}{3} \Delta g-\delta g\langle\cos 2 \phi\rangle
\end{aligned}
$$

These approximations are deduced from Eq. (21) assuming that the $z$-axis is highly ordered. The $\left\langle g_{x x}\right\rangle,\left\langle g_{y y}\right\rangle$ elements are then primarily sensitive to rotation about the chain long axis and the $\left\langle g_{z z}\right\rangle$ element to off-axis excursions (see Fig. 1). More exact expressions can be found in Kurad et al. (2001).

Fig. 4 gives the dependence of $\left\langle g_{x x}\right\rangle,\left\langle g_{y y}\right\rangle$ and $\left\langle g_{z z}\right\rangle$ on position of chain labelling in the cholesterol-containing liquid-ordered membranes. The profile of non-axial ordering exhibited by $\left\langle g_{x x}\right\rangle$ and $\left\langle g_{y y}\right\rangle$ corresponds to the expected location of cholesterol within the membrane. If the $3 \beta-\mathrm{OH}$ group is situated at the polar-apolar interface, then the rigid steroid nucleus of cholesterol extends to approximately C-atom $n=11$ of the $s n-2$ chain. The lateral ordering of the lipid chains, characterised by the large and approximately con-

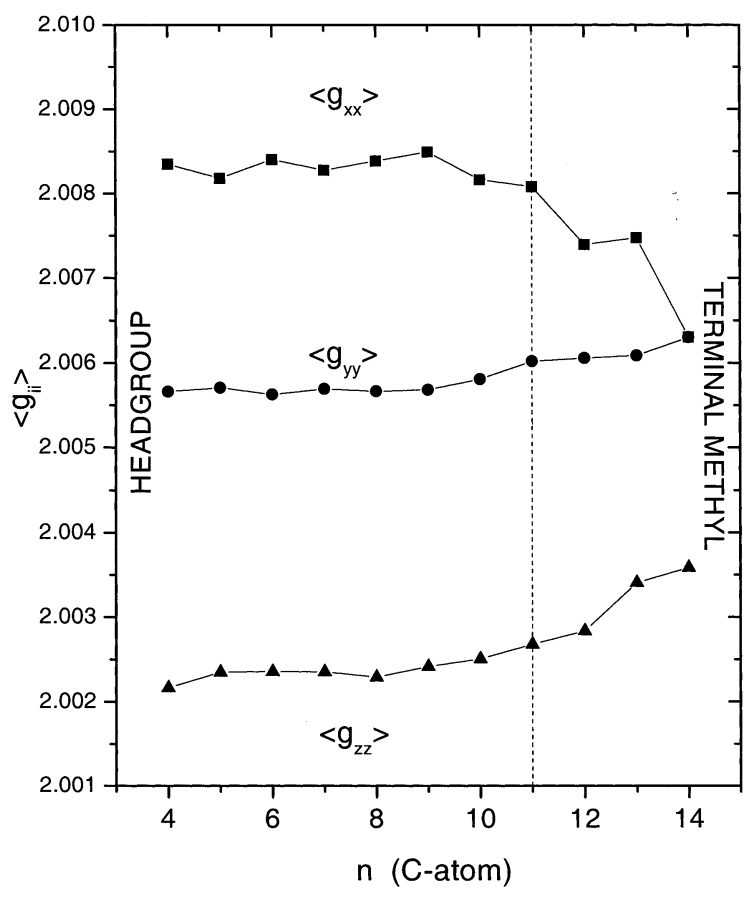

Fig. 4. Effective $g$-tensor elements $\left\langle g_{x x}\right\rangle,\left\langle g_{y y}\right\rangle$ and $\left\langle g_{z z}\right\rangle$ as a function of spin-label position, $n$, in the $s n-2$ chain of phosphatidylcholine for membranes of dimyristoyl phosphatidylcholine-cholesterol $(3: 2 \mathrm{~mol} / \mathrm{mol})$ at $30{ }^{\circ} \mathrm{C}$ (Kurad et al., 2001).

stant $\left\langle g_{x x}\right\rangle-\left\langle g_{y y}\right\rangle$ anisotropy is therefore induced by the sterol nucleus. Beyond this, axial rotation rapidly sets in and is coupled to an increase in the amplitude of segmental off-axis motion that is seen from the values of $\left\langle g_{z z}\right\rangle$ (and is also manifested in the asymmetric motional averaging of $\left\langle g_{x x}\right\rangle$ and $\left\langle g_{y y}\right\rangle$ - see Kurad et al., 2001).

The lateral ordering of the lipid molecules by cholesterol in liquid-ordered phases could be a mechanism for the formation of segregated inplane membrane domains. Existence of the latter has been deduced indirectly from studies of detergent-insoluble cell membrane fractions that are rich in cholesterol, sphingolipids and GPI-anchored proteins (Brown and Rose, 1992). The liquid-ordered phase has been implicated in such putative sphingolipid rafts that are proposed as vehicles for intracellular membrane sorting and a variety of cellular signalling processes (Simons and Ikonen, 1997). 


\section{Spectral simulations for non-axiality}

Resolution of the $g_{x x}$ and $g_{y y}$ features in highfield spectra may, in principle, be the result of two rather different modes of rotational motion. This depends on whether rotation is in the fast or slow regime and has very distinct consequences for the interpretation in terms of molecular interactions. Relatively rapid nonaxial $\phi$-rotation of restricted angular amplitude leads to only partial averaging of the $g_{x x}-g_{y y}$ tensor anisotropy. This situation of in-plane ordering is that considered for cholesterol interactions in the preceding section. Alternatively, slow but unrestricted axial rotation in the $x-y$ plane, with a correlation time such that $\tau_{\mathrm{R} \|} \gg \hbar /\left(g_{x x}-g_{y y}\right) \beta_{\mathrm{e}} H$ (cf. Table 3 ), could also give rise to incomplete averaging of the $g_{x x}-g_{y y}$ anisotropy. In this latter case, there is no preferential in-plane orientation; the $x-y$ anisotropy depends solely on the rate of motion.

Discussion has already been given of the distinction between slow unrestricted motion and rapid 'librational' motion of limited amplitude (see Table 5). In principle, these features can be used to distinguish the two possible origins of $g_{x x}-g_{y y}$ anisotropy. Rapid rotation of limited amplitude gives rise primarily to line shifts, whereas slow unrestricted axial rotation is accompanied also by appreciable line broadening. In practice, other sources of linebroadening - particularly from off-axial motions - can make the distinction not so clear cut. Therefore in more complex situations spectral simulation is required.

High-field spin-label simulations directed particularly at the non-axial lipid ordering by cholesterol have been performed by Livshits and Marsh (2000). Two relatively simple models were used to keep the number of free parameters to a minimum consistent with adequately distinguishing between the two physical cases. These are fast-motional and slow-motional models, respectively.

\subsection{Rapid rotations of limited amplitude}

The fast-motional model (Israelachvili et al., 1975) uses perturbation theory to describe relatively rapid motions that are restricted in angular amplitude both of off-axis motion $(\theta)$ of the nitroxide $z$-axis and of $\phi$-rotation around the $z$-axis (i.e., the $x-y$ amplitude). It is assumed that the angular excursions of the nitroxide $z$-axis are confined to a cone of angle $\theta=\theta_{\mathrm{o}}$, and that the maximum azimuthal excursions of the nitroxide $x$ - (or $y$-) axis about the $z$-axis are given by $\phi=\phi_{\mathrm{o}}$. Within these angular ranges, the probability of a particular orientation $(\theta, \phi)$ is taken to be random (i.e., the usual $\sin \theta$ weighting for the $\theta$-distribution). The motionally averaged spectral line positions $\left\langle H_{m_{I}}\left(\theta_{\mathrm{o}}, \phi_{\mathrm{o}}\right)\right\rangle$ are then given by Eq. (21), where the required angular averages are presented in Israelachvili et al. (1975). Time-dependent perturbation theory then gives the rotational Lorentzian linewidths as:

$\frac{1}{T_{2, m_{I}}}=\gamma_{\mathrm{e}}^{2}\left\langle\left[H_{m_{I}}(\theta, \phi)-\left\langle H_{m_{I}}\left(\theta_{\mathrm{o}}, \phi_{\mathrm{o}}\right)\right\rangle\right]^{2}\right\rangle \tau_{\mathrm{R}}$

where $\tau_{\mathrm{R}}$ is the rotational correlation time that is assumed for simplicity to be the same for axial and off-axis rotations. In Eq. (25), $H_{m_{I}}(\theta, \phi)$ is the instantaneous resonance position for a general orientation $\theta, \phi$ of the magnetic field. It is given by Eq. (21) without angular brackets. The outer angular brackets in Eq. (25) represent a further angular average over the orientational ranges $\theta=$ 0 to $\theta_{\mathrm{o}}$ and $\phi=0$ to $\pm \phi_{\mathrm{o}}$. This requires evaluation of higher angular averages, e.g. $\left\langle\cos ^{4} \theta\right\rangle$, which are also given in Israelachvili et al. (1975).

Fig. 5 (left-hand column) gives simulations for increasing amplitudes $\phi_{\mathrm{o}}$ of axial rotation about a fixed $z$-axis (i.e., $\theta_{\mathrm{o}}=0$ ). The fast motional formalism is used with a fixed axial rotational rate of $\tau_{\mathrm{R} \|}^{-1}=6 \times 10^{8} \mathrm{~s}^{-1}$. The spin Hamiltonian parameters and residual linewidths used in the simulation are those representative of DOXYL lipid spin labels in membranes (cf. Table 2). Correspondingly, the low-field peak is relatively broad, because of $g_{x x}$-strain that arises from inhomogeneities in the local environmental polarity (see later sections). Increasing amplitudes of axial rotation progressively average the anisotropy between the $g_{x x}$ and $g_{y y}$ canonical regions of the spectrum. Essentially complete averaging is obtained for $\phi_{\mathrm{o}} \approx 90^{\circ}$. The width of the resulting single, first-derivative like feature in the $g_{x x}, g_{y y}$ region is then determined by the rotational rate $\tau_{\mathrm{R} \|}^{-1}$ (see Eq. (25)). 
7.2. Axially anisotropic jumps of unrestricted frequency

The model chosen as particularly appropriate to slow motions involves uncorrelated jumps of the nitroxide $z$-axis restricted within a cone of angle $\theta_{\mathrm{o}}$ and rotational jumps around the $z$-axis over the full angular range $\phi=0-2 \pi$. This is an extension of the symmetric-top jump model of Livshits (1976) to anisotropic media. The Bloch equations for the magnetization components $\mathbf{M}(u$, v) are written as (Livshits, 1976):

$$
\begin{aligned}
& \frac{\mathrm{d} \mathbf{M}}{\mathrm{d} t}+\left(A+\tau_{\mathrm{R} \|}^{-1} \mathbf{E}\right) \mathbf{M} \\
& =\tau_{\mathrm{R} \perp}^{-1} \int_{0}^{\theta_{\mathrm{O}}} \int_{o}^{2 \pi} \mathbf{M} \sin \theta \mathrm{d} \theta \mathrm{d} \phi \\
& \quad+\left(\tau_{\mathrm{R} \|}^{-1}-\tau_{\mathrm{R} \perp}^{-1}\right) \int_{\mathrm{o}}^{2 \pi} \mathbf{M ~ d} \phi+\mathbf{M}_{\mathrm{o}}
\end{aligned}
$$

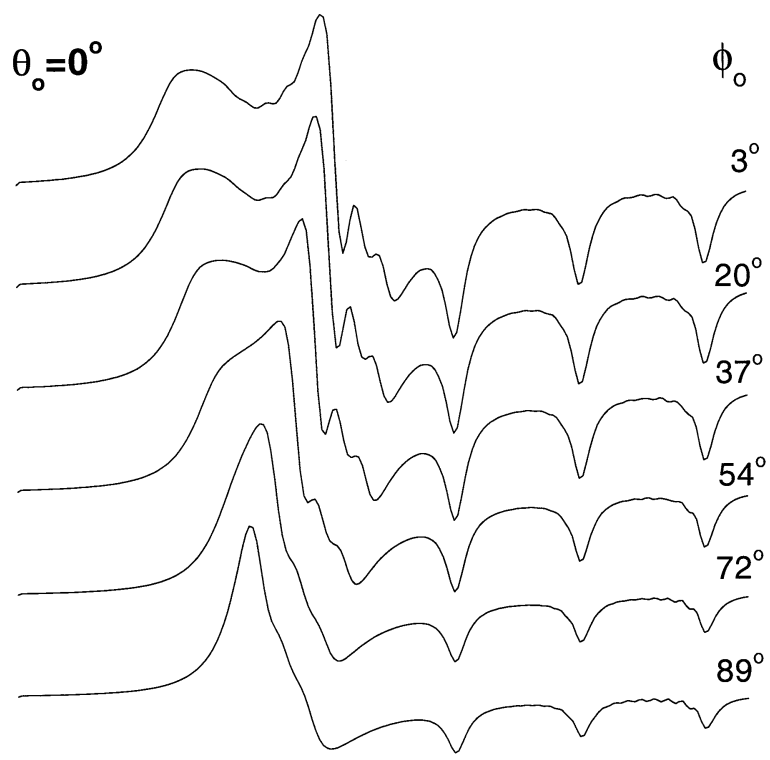

$$
\tau_{\mathrm{R} / /}^{-1}=6 \times 10^{8} \mathrm{~s}^{-1}
$$

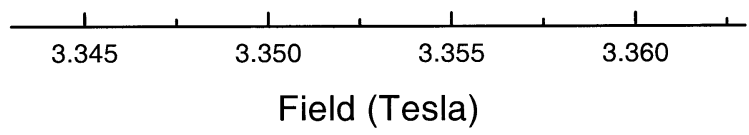

where $\mathbf{E}$ is the unit matrix, the static magnetization is $\mathbf{M}_{\mathrm{o}}=\left(0, M_{\mathrm{o}}\right)$, and $\tau_{\mathrm{R} \|}^{-1}, \tau_{\mathrm{R} \perp}^{-1}$ are the jump rates for rotation around the $z$-axis and for rotation of the long axis, respectively. The Bloch matrix in Eq. (26) is defined by:

$$
\mathbf{A}=\left(\begin{array}{cc}
T_{2,0}^{-1} & \gamma_{\mathrm{e}}\left[H-H_{m_{I}}(\theta, \phi)\right] \\
-\gamma_{\mathrm{e}}\left[H-H_{m_{I}}(\theta, \phi)\right] & T_{2,0}^{-1}
\end{array}\right)
$$

where $H_{m_{I}}(\theta, \phi)$ is, as above, the general resonance position, $H$ is the field coordinate for the lineshape, and $T_{2,0}^{-1}$ represents the intrinsic linewidth. This model implies unrestricted uniaxial rotation over $\phi$ with a frequency $\tau_{\mathrm{R} \|}^{-1}$, and limited rotation over the angle $\theta$ from 0 to $\theta_{\mathrm{o}}$ with frequency $\tau_{\mathrm{R} \perp}^{-1}$. The steady-state solutions $(\mathrm{d} \mathbf{M} /$ $\mathrm{d} t=0)$ of Eq. (26) give the lineshapes $v\left(H, H_{m_{I}}\right)$ of the ESR absorption corresponding to the individual ${ }^{14} \mathrm{~N}$-hyperfine states. Dispersion contribu-

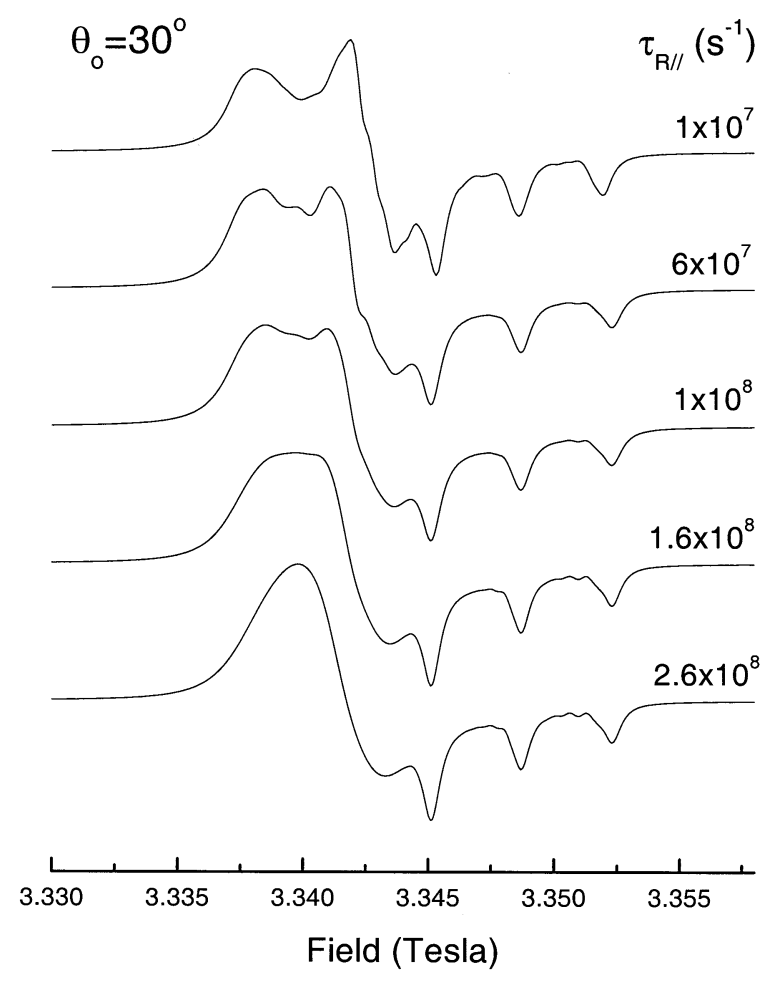

Fig. 5. $94 \mathrm{GHz}$ spin-label ESR spectra, simulated using motional narrowing theory (left column) and an anisotropic random jump model (right column). Left-hand spectra correspond to rapid rotation of increasing amplitude $\phi_{\mathrm{o}}$ about the $z$-axis at a fixed rate of $\tau_{\mathrm{R}}^{-1}=6 \times 10^{8} \mathrm{~s}^{-1}$. Right-hand spectra correspond to fully axial jump reorientation of increasing rate $\tau_{\mathrm{R}}^{-1}$, for a restricted off-axis amplitude of angle $\theta_{\mathrm{o}}=30^{\circ}$ with fixed overall rotational rate of $\tau_{\mathrm{R}}^{-1}=10^{7} \mathrm{~s}^{-1}$ (Livshits and Marsh, 2000). 
tions are given by the corresponding $u\left(H, H_{m_{I}}\right)$ solutions. The latter may be needed for highfield lineshape simulations, because dispersion admixture is sometimes unavoidable with aqueous samples.

Fig. 5 (right-hand column) gives simulations for a fixed amplitude and rate of off-axis rotation, with increasing rates of full axial rotation. The latter extend from the slow-motional $\left(\tau_{\mathrm{R} \|}^{-1} \sim 10^{7} \mathrm{~s}^{-1}\right)$ into the fast motional $\left(\tau_{\mathrm{R} \|}^{-1} \sim\right.$ $\left.3 \times 10^{8} \mathrm{~s}^{-1}\right)$ regime. Spin Hamiltonian parameters and residual linewidths are the same as those used for the previous simulations. As the rate of axial rotation increases, the separation of the $g_{x x}$ - and $g_{y y}$-spectral features gradually decreases. At sufficiently high axial rotation frequencies, the $g_{x x}-g_{y y}$ anisotropy becomes completely averaged.

Comparison of the left- and right-hand panels of Fig. 5 suggests that the differential patterns of line broadening that accompany loss of the $g_{x x}-g_{y y}$ anisotropy should be sufficient to distinguish between the two models. Direct simulation of the experimental $94 \mathrm{GHz}$ spectra from high-cholesterol membranes of the type given in Fig. 3 that the non-axial $x-y$ anisotropy is a consequence of strictly limited rotation about the lipid chain axis, i.e., of lateral ordering of the lipid molecules in the membrane plane (Livshits and Marsh, 2000). Simulations for unrestricted azimuthal rotation do not fit the spectra so well, indicating that resolution of the $x-y$ features is not a result of the spectra being driven into the slow-motion regime by the high fields of $94-\mathrm{GHz}$ ESR. In contrast, almost complete and relatively rapid axial motion was found from simulations (Livshits and Marsh, 2000) of 94-GHz spectra from spin-labelled fatty acids in phosphatidylcholine membranes not containing cholesterol (Smirnov et al., 1998). This demonstrates that lateral ordering of the lipids is a particular feature of the liquid-ordered phase of cholesterol-containing membranes.

\section{Aligned membranes and biaxiality}

The physical size of oriented samples poses problems at high microwave frequencies, and they can only be accommodated in quasi-optical, etalon-type resonators. One such holder for aqueous samples is described by Barnes and Freed (1997). Changing the sample orientation relative to the static field of the superconducting magnet also requires modification of the resonator configuration from a normal transmission-mode etalon to a 'shunt' Fabry-Perot resonator (Barnes and Freed, 1998a,b).

Barnes and Freed (1998a) have examined the spin-labelled steroid cholestane (CSL) in oriented membranes of dimyristoyl phosphatidylcholine (DMPC) in the gel phase by using $250 \mathrm{GHz}$ ESR. This particular spin label has the nitroxide $y$-axis directed almost parallel to the long molecular axis of the steroid molecule. With the magnetic field parallel to the bilayer normal, a single first-derivative like resonance is observed at the position corresponding to $g_{y y}$. With the magnetic field lying within the plane of the membrane, the spectrum is a two-dimensional powder spectrum. The extrema in the anisotropic powder pattern correspond to the $g_{x x}$ and $g_{z z}$ positions, with no features visible at the $g_{y y}$ position. The cholestane spin label is therefore oriented with its long axis almost parallel to the membrane normal in the DMPC gel-phase and is not rotating rapidly about this axis on the $250-\mathrm{GHz}$ ESR timescale. This confirms previous similar studies at $9 \mathrm{GHz}$ (Marsh, 1980), where it was shown that rapid rotation about the long axis of CSL sets in first at the pretransition of DMPC.

Extraordinary results were obtained when similar 250-GHz ESR experiments were performed on aligned bilayers of dimyristoyl phosphatidylserine (DMPS) (Barnes and Freed, 1998a). For the magnetic field parallel to the membrane normal, a broad first-derivative spectrum was found centred at the midpoint between the $g_{y y}$ and $g_{z z}$ positions. For the magnetic field in the plane of the membrane, a two-dimensional powder pattern with rather broad lines was observed. Unlike for DMPC, this powder pattern extended from the $g_{x x}$ position only as far as the $(1 / 2)\left(g_{y y}+g_{z z}\right)$ midpoint. The implications of these results are that, as in DMPC, the cholestane spin label does not rotate rapidly 
about its long axis in gel-phase DMPS. However, in DMPS, CSL reorients relatively rapidly about the $x$-axis which is normal to the plane of the steroid nucleus. This causes the long axis of the steroid to exchange between orientations parallel and perpendicular to the membrane normal. Such a strongly pronounced biaxiality in the ordering of an amphiphilic lipid in bilayer membranes is practically unprecedented, and, as already described, is not found for CSL in the gel-phase DMPC host.

\section{Polarity dependence of hyperfine splitting}

The conventional method of studying environmental polarity with nitroxide spin labels is via the overall magnitude of the hyperfine splittings. This aspect is considered here as an introduction to studies on the polarity dependence of the $g$-tensor by high-field ESR. In addition, advances have been made recently in the study of the polarity profile in lipid bilayer membranes by using conventional low-field spin label spectroscopy. It is therefore appropriate to discuss these in the present volume.

The polarity dependence of the ${ }^{14} \mathrm{~N}$-hyperfine splitting is usually characterised either by the isotropic hyperfine splitting constant, $a_{\mathrm{o}}$, or by the rigid-limit hyperfine tensor element, $A_{z z}$. The isotropic hyperfine splitting, $a_{\mathrm{o}}^{\mathrm{N}}$, arises from polarisation of the electron spin density at the nitrogen nucleus by the unpaired electron in the free radical $2 p \pi$-orbital. It is related to the unpaired electron densities, $\rho_{\mathrm{N}}$ and $\rho_{\mathrm{O}}$ in the $2 p \pi$-orbital at the nitrogen and oxygen atoms, respectively, by a McConnell-type relation (Karplus and Fraenkel, 1961):

$a_{\mathrm{o}}^{\mathrm{N}}=Q_{\mathrm{N}} \rho_{\mathrm{N}}+Q_{\mathrm{O}} \rho_{\mathrm{O}}$

where $Q_{\mathrm{N}} \approx 2.42 \mathrm{mT}$ and $Q_{\mathrm{O}} \approx 0.36 \mathrm{mT}$ (Cohen and Hoffman, 1973). Because $\rho_{\mathrm{N}}+\rho_{\mathrm{O}}=1$, the isotropic splitting is given by $a_{\mathrm{o}}^{\mathrm{N}} \approx\left(Q_{\mathrm{N}}-\right.$ $\left.Q_{\mathrm{O}}\right) \rho_{\mathrm{N}}+Q_{\mathrm{O}}$ and is directly proportional to the spin density on the nitrogen. Typically $a_{o}^{\mathrm{N}} \sim 1.4-$ $1.6 \mathrm{mT}$ for a nitroxide, which implies $\rho_{\mathrm{N}} \sim 0.5$ 0.6 . This value depends, however, on the polarity of the environment. The polarity dependence of the hyperfine splittings arises from shifts in the unpaired spin density between the two canonical structures:

$>\mathrm{N}-\mathrm{O}^{\bullet} \leftrightarrow>\mathrm{N}^{+}-\mathrm{O}^{-}$

where the right-hand structure is favoured by a polar environment and disfavoured by an apolar environment. It has the unpaired electron located on the nitrogen and therefore polar environments result in larger values of $a_{\mathrm{o}}^{\mathrm{N}}$.

The $A_{z z}$ hyperfine tensor element is composed of an isotropic and an anisotropic part: $A_{z z}=$ $a_{\mathrm{o}}+(2 / 3) \Delta A$. The anisotropic part arises from a magnetic dipolar interaction between the unpaired electron spin and the nitrogen nuclear spin. Therefore it also depends on $\rho_{\mathrm{N}}$, the unpaired electron density on the nitrogen. The result is that the polarity dependence of $A_{z z}$ is greater than that of $a_{\mathrm{o}}^{\mathrm{N}}$. For DOXYL nitroxides, the relation established experimentally between the two in solvents of different polarity is (Marsh, 1981; Subczynski et al., 1994):

$A_{z z}^{\mathrm{R}}=2.35 \times a_{\mathrm{o}}^{\mathrm{N}}-0.084 \mathrm{mT}$

For homogeneous solvent systems, the environmental polarity is likely to be the same for the liquid state in which $a_{\mathrm{o}}^{\mathrm{N}}$ is measured as for the frozen state in which the rigid-limit value of $A_{z z}$ is measured. In the latter case, it is necessary to choose solvent systems that form rigid glasses. For inhomogeneous systems such as membranes, environmental polarities in the fluid and rigid states might not be identical. For instance, water may be squeezed out from the interior of frozen membranes.

Stabilisation of the polar canonical form of the nitroxide is determined by the strength of the polarising or 'reaction' field of the solvent. This field is established by local polarisation of the solvent that is induced by the dipole moment of the nitroxide. For aprotic (i.e., non-H-bonding) solvents, this gives rise to a predictable dependence of $a_{\mathrm{o}}^{\mathrm{N}}$ on the dielectric constant of the solvent (Griffith et al., 1974; Seelig et al., 1972). In hydrogen-bonding solvents, the values of $a_{\mathrm{o}}^{\mathrm{N}}$ are greater than would be predicted from the dependence on dielectric constant. Experiments in mixed solvent systems reveal that the additional 
increase in $a_{\mathrm{o}}^{\mathrm{N}}$ is linearly proportional to the concentration of $\mathrm{H}$-bond donor. In general, the complete dependence of the isotropic hyperfine splitting on polarity is given by (Gagua et al., 1978; Marsh, 1981):

$a_{\mathrm{o}}^{\mathrm{N}}=a_{\mathrm{o}}^{\varepsilon=1}+K_{v} \frac{\varepsilon-1}{\varepsilon+1}+K_{\mathrm{h}} p$

where $\varepsilon$ is the dielectric constant of the medium and $p$ is the concentration of $\mathrm{H}$-bond donor. The strengths of the reaction-field and hydrogen-bonding contributions are specified by $K_{v}$ and $K_{h}$, respectively. For DOXYL spin labels of the type used in spin-labelled lipid probes: $a_{\mathrm{o}}^{\varepsilon=}$ $1=1.385 \pm 0.009 \mathrm{mT}$ and $K_{v}=0.064 \mathrm{mT}$ (Marsh, 1981).

\section{Polarity profile of lipid membranes}

The environmental dependence of the isotropic hyperfine splitting, described by Eq. (30) in the previous section, can be used to determine the profile of polarity and water permeation across lipid bilayer membranes. Results established for bilayer membranes of dipalmitoyl phosphatidylcholine with and without equimolar cholesterol are given in Fig. 6 (Marsh, 2001). Isotropic hyperfine splittings, $a_{\mathrm{o}}^{\mathrm{N}}$, were determined as a function of labelling position in the $s n-2$ acyl chain of $s n-2-(n$-DOXYL-stearoyl) phosphatidylcholine spin labels. Measurements were made at temperatures in the fluid membrane phase. For axially anisotropic spectra, the isotropic hyperfine splitting was calculated from the trace: $a_{\mathrm{o}}^{\mathrm{N}}=(1 / 3)\left(A_{\|}+2 A_{\perp}\right)$, where $A_{\|}$and $A_{\perp}$ are the hyperfine splitting constants for the magnetic field oriented parallel and perpendicular, respectively, to the membrane normal. For spin labels close to the terminal methyl group of the chains, $a_{\mathrm{o}}^{\mathrm{N}}$ was measured directly from truly isotropic spectra recorded at high temperature. In both cases, lack of appreciable temperature dependence was used as a criterion to discount artefacts from slow motion or residual anisotropy, respectively.

The $a_{\mathrm{o}}$-profile given in Fig. 6 is mirrored about the membrane mid-plane, thus reflecting

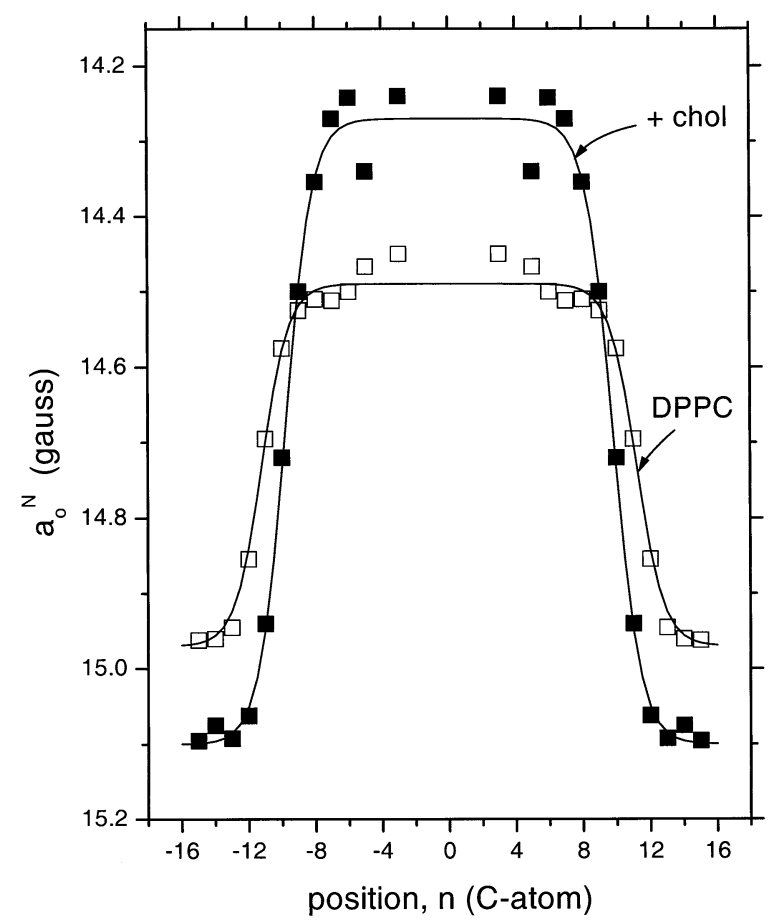

Fig. 6. Polarity profiles of the isotropic ${ }^{14} \mathrm{~N}$-hyperfine splitting constant, $a_{\mathrm{o}}^{\mathrm{N}}$, of $n$-DOXYL phosphatidylcholine spin labels in fluid bilayer membranes of dipalmitoyl phosphatidylcholine without (open squares) and with (solid squares) $50 \mathrm{~mol} \%$ cholesterol. Data are reflected about the predicted bilayer mid-plane $\left(n-n_{c}=0 ; n_{c}=19\right)$, and are plotted with decreasing $a_{\mathrm{o}}^{\mathrm{N}}$, i.e. as the hydrophobic barrier. Lines are fits of the profile given by Eq. (31). Data from (Marsh, 2001).

the trans-bilayer symmetry. A sigmoidal troughlike polarity profile represents the membrane hydrophobic barrier to permeation of water and polar solutes (for this reason the ordinate corresponds to decreasing $a_{\mathrm{o}}^{\mathrm{N}}$ in Fig. 6). It has the so-called Boltzmann sigmoidal form that can be described for each bilayer half by (Marsh, 2001):

$a_{\mathrm{o}}^{\mathrm{N}}(n)=\frac{a_{\mathrm{o}, 1}^{\mathrm{N}}-a_{\mathrm{o}, 2}^{\mathrm{N}}}{1+\mathrm{e}^{\left(n-n_{\mathrm{o}}\right) / \lambda}}+a_{\mathrm{o}, 2}^{\mathrm{N}}$

where $a_{\mathrm{o}, 1}^{\mathrm{N}}$ and $a_{\mathrm{o}, 2}^{\mathrm{N}}$ are the limiting values of the isotropic hyperfine splitting constant before and after the barrier, $n_{\mathrm{o}}$ is the midpoint at which $a_{\mathrm{o}}^{\mathrm{N}}\left(n_{\mathrm{o}}\right)=(1 / 2)\left(a_{0,1}^{\mathrm{N}}+a_{\mathrm{o}, 2}^{\mathrm{N}}\right)$, and $\lambda$ determines the width of the transition region. Eq. (31) has a 
straightforward statistical thermodynamic interpretation. It represents a distribution between the two regions $n<n_{\mathrm{o}}$ and $n>n_{\mathrm{o}}$, where the distributional free energy changes linearly with distance into each region. It seems reasonable to identify these two regions with the interfacial and hydrophobic core regions of the membrane defined with liquid-state diffraction techniques by Wiener and White, (1992).

Typically the position of the dividing surface is specified by $n_{\mathrm{o}} \sim 8$ and the thickness of the transition region by $\lambda \sim 1 \mathrm{CH}_{2}$ unit (Marsh, 2001). The presence of equimolar cholesterol increases $n_{\mathrm{o}}$ by approximately $1 \mathrm{CH}_{2}$ unit, but its most pronounced effect is in increasing the height of the hydrophobic barrier. The values of $a_{\mathrm{o}, 1}^{\mathrm{N}}$ are increased somewhat to more polar values by incorporation of cholesterol, and those of $a_{\mathrm{o}, 2}^{\mathrm{N}}$ are decreased to values close to those for a pure hydrocarbon solvent. Whereas there are non-vanishing amounts of water in the interior of fluid lipid membranes, equimolar cholesterol reduces the level to practically zero at the centre of the membrane.

Having defined the permeation barriers in terms of Eq. (31) it is then possible to evaluate their contribution to the overall membrane permeability and the modification of this by cholesterol (Marsh, 2001). The permeability barrier is given by (Diamond and Katz, 1974):

$\delta\left(\frac{1}{P}\right)=\int_{0}^{2 d} \frac{\mathrm{d} x}{K(x) D(x)}$

where $P$ is the permeability coefficient, $K(x)$ and $D(x)$ are the local partition coefficient and diffusion coefficient of the permeant species at position $x$ into the membrane, and $2 d$ is the diffusive thickness of the membrane. From the dependence of the polarity profile on local water concentration that is given by the term $K_{\mathrm{h}} p$ in Eq. (30), it is reasonable to assume that the product $K(x) D(x)$ for water has the same transverse profile as that given by Eq. (31) (see Marsh, 2001).

Performing the integration in Eq. (32) then yields the following contribution to the permeability barrier:

$$
\begin{aligned}
\delta\left(\frac{1}{P}\right) & \\
= & \frac{2}{K_{1} D_{1}}\left[d+\lambda\left(\frac{K_{1} D_{1}}{K_{2} D_{2}}-1\right)\right. \\
& \left.\times \ln \left(\frac{K_{1} D_{1} / K_{2} D_{2}+\mathrm{e}^{\left(d-d_{\mathrm{o}}\right) / \lambda}}{K_{1} D_{1} / K_{2} D_{2}+\mathrm{e}^{-d_{\mathrm{o}} / \lambda}}\right)\right]
\end{aligned}
$$

where $d_{\mathrm{o}}$ is the transverse distance corresponding to chain position $n_{\mathrm{o}}$, and $\lambda$ is also expressed as a distance. $K_{1} D_{1}$ and $K_{2} D_{2}$ are the partition coefficient and diffusion coefficient products at the beginning of the apolar region and at the centre of the membrane, respectively. The first term in Eq. (33) is the normal diffusive resistance of a membrane of thickness $2 d$ with solute partition and diffusion coefficients of $K_{1}$ and $D_{1}$, respectively. The second term is the additional resistance caused by the permeability barrier. It can be viewed as an effective increase in membrane diffusive thickness, which from the data of Fig. 6 corresponds to approximately $12 \mathrm{CH}_{2}$ segments for dipalmitoyl phosphatidylcholine membranes. Assuming the barrier to be rate limiting, equimolar cholesterol is correspondingly predicted to decrease water permeability by approximately 50\%, in agreement with experiment (Marsh, 2001).

Interestingly, permeation profiles for oxygen, which is a small apolar solute, are similar to those for water, except that they are understandably of the opposite sense (see Marsh, 2001). Oxygen profiles, which rigorously measure the $K(x) D(x)$ concentration-diffusion product (Subczynski et al., 1989), can be determined from spin-lattice relaxation enhancement experiments with spin-labelled lipids (see also Marsh et al., 1998).

\section{Polarity dependence of the g-tensor}

Polarity effects on the $g$-tensor first become of critical importance in high-field ESR. The polarity dependence of the $g$-values for nitroxide spin labels has been analysed by Kawamura et al. (1967). They used the theory of Stone (1963) for the $g$-tensor of $\pi$-radicals. The second-order perturbation expression for a particular diagonal element, $g_{i i}$, of the $g$-tensor involves cross terms 
between the magnetic field interaction and the spin-orbit coupling and is given by:

$g_{i i}=g_{\mathrm{e}}+2 \sum_{m \neq p} \frac{\sum_{k, k^{\prime}}\left\langle\chi_{p}^{\left(k^{\prime}\right)}\left|l_{i}^{\left(k^{\prime}\right)}\right| \chi_{m}^{\left(k^{\prime}\right)}\right\rangle \zeta_{k}\left\langle\chi_{m}^{(k)}\left|l_{i}^{(k)}\right| \chi_{p}^{(k)}\right\rangle}{E_{p}-E_{m}}$

where $p$ represents the unpaired electron orbital and $m$ the other occupied and unoccupied orbitals. For nitroxide spin labels the unpaired electron is located in a $2 p_{z} \pi$ orbital, and the other important orbitals are the bonding and antibonding $\sigma$-orbitals ( $b$ and $a$ ) of the $\mathrm{N}-\mathrm{O}$ bond and the lone pair orbitals $(n)$ on the oxygen. The superscripts $k, k^{\prime}$ refer to the atom centres $N$ and $O$, and $\zeta_{k}$ is the corresponding spin-orbit coupling constant. For example, $\chi_{m}^{(k)}$ is the contribution of the atomic orbital from atom $k$ to the molecular orbital $m$; the coefficient giving the weighting for this atomic orbital is designated $C_{k}^{(m)}$.

For a nitroxide spin label, the bonding and antibonding $\mathrm{N}-\mathrm{O} \sigma$-orbitals are composed of linear combinations of $2 s$ and $2 p_{x}$ orbitals. From Eq. (34) it therefore follows that the $\mathrm{N}-\mathrm{O} \sigma$-bond contributes only to the $g_{y y}$-element of the $g$-tensor. If the lone pair is confined to a $2 p_{y}$ orbital on the oxygen, this then contributes only to the $g_{x x}$-element of the $g$-tensor. Under these circumstances the elements of the $g$-tensor are given by (Kawamura et al., 1967):

$g_{x x}=g_{\mathrm{e}}+\frac{2 \zeta_{\mathrm{O}}\left(C_{\mathrm{O}, y}^{(n)}\right)^{2} \rho_{\mathrm{O}}}{E_{p}-E_{n}}$

$g_{y y}=g_{\mathrm{e}}+2 \sum_{m}^{a, b}$

$\frac{\zeta_{\mathrm{N}}\left(C_{\mathrm{N}, x}^{(m)}\right)^{2} \rho_{\mathrm{N}}+\zeta_{\mathrm{O}}\left(C_{\mathrm{O}, x}^{(m)}\right)^{2} \rho_{\mathrm{O}}+\left(\zeta_{\mathrm{N}}+\zeta_{\mathrm{O}}\right) C_{\mathrm{N}, x}^{(m)} C_{\mathrm{O}, x}^{(m)} p_{\mathrm{NO}}}{E_{p}-E_{m}}$

$g_{z z}=g_{\mathrm{e}}$

where $g_{\mathrm{e}}(=2.00232)$ is the free electron $g$-value; $C_{\mathrm{N}, x}^{(b)}, C_{\mathrm{N}, x}^{(a)}$ are coefficients of the nitrogen $2 p_{x}$ orbital in the bonding and antibonding $\mathrm{N}-\mathrm{O} \sigma$-orbitals; $C_{\mathrm{O}, y}^{(n)}$ is the coefficient of the oxygen $2 p_{y}$ orbital in the lone pair orbital, etc. As usual, $\rho_{\mathrm{N}}$ and $\rho_{\mathrm{O}}$ are the unpaired electron densities on the nitrogen and oxygen atoms, respectively, and $p_{\mathrm{NO}}$ is the partial bond order of the unpaired electron orbital. If the lone pair orbital is an $\mathrm{sp}^{2}$ hybrid, the above is modified only in that the lone pair then contributes also to the $g_{y y}$ element, as well as to $g_{x x}$. A term similar to that in Eq. (35), but involving the $C_{\mathrm{O}, x}^{(n)}$ admixture coefficient, is then added to the right-hand side of Eq. (36). Recent molecular orbital calculations, however, predict practically pure unhybridised $2 p_{y}$-orbitals for the oxygen lonepair electrons (Steinhoff et al., 2000).

Comparison of the expressions given by Eqs. (35)-(37) with the experimental $g$-tensors reported in Table 2, reveals, as predicted, that it is the $g_{z z}$ element which has the smallest value, closest to that of a free electron. The largest deviation from the free electron $g$-value is for the $g_{x x}$-element. This implies that the contribution to the $g$-value from the lone pair orbital is greater than that from the $\mathrm{N}-\mathrm{O}$ bond (which contributes only to $g_{y y}$ ). This fulfills the expectation that $C_{\mathrm{O}, y}^{(n)} \approx 1$ and that the lone-pair lies closer in energy to the unpaired electron orbital than does the $\mathrm{N}-\mathrm{O} \sigma$-bonding orbital. Potentially, the solvent dependence of the $g$-tensor can be greater than that of the hyperfine tensor. As seen previously, the latter depends only on the distribution of unpaired electron density, $\rho_{\mathrm{N}}$. The polarity shifts in $g$-tensor, however, depend additionally on lowering of the lone-pair orbital energy, $E_{n}$ (indicated by the blue shift in the $n-\pi^{*}$ optical transition), as well as on delocalisation of the lone pair orbital (via the $C_{\mathrm{O}, y}^{(n)}$ coefficient).

Experimentally, it is found that the $g_{x x}$-tensor element displays by far the largest polarity dependence and that of $g_{z z}$ is rather small (Ondar et al., 1985). Some representative measurements of $g_{x x}$ in glassy solvents of different polarities are shown in Fig. 7. As the $A_{z z}$ hyperfine tensor element increases in more polar media (because, it is directly proportional to $\rho_{\mathrm{N}}$ ), the $g_{x x}$-tensor element progressively decreases, because it depends on $\rho_{\mathrm{O}}=1-\rho_{\mathrm{N}}$. Data obtained at $250 \mathrm{GHz}$ for DOXYL spin-labelled lipids in frozen hydrated dipalmitoyl phosphatidylcholine bilayer membranes are also given in Fig. 7. A subset of the original data that does not display pronounced spin-spin broadening (i.e., spin label segregation) is presented in this figure. The absolute values of $g_{x x}$ are different for the DOXYL spin labels than for the piperidine-based (TEMPO) spin label. However, with differing polarity according to 
spin-label chain position, they display a very similar dependence on $A_{z z}$ to that of the TEMPOL spin label in glassy solvents.

A common feature of high-field spectra, such as the upper ones in Fig. 3, is the greater width of the $g_{x x}$ feature than that of the $g_{y y}$ or $g_{z z}$ features. This is almost certainly linked to the greater polarity sensitivity of $g_{x x}$ relative to that of the other $g$-tensor elements. The additional broadening, or $g$-strain, represents a heterogeneity in polarity of the environment in which the spin label is located. Potentially, measurements of this type with spin-labelled lipids can provide more detailed information on the distribution of water in membranes.

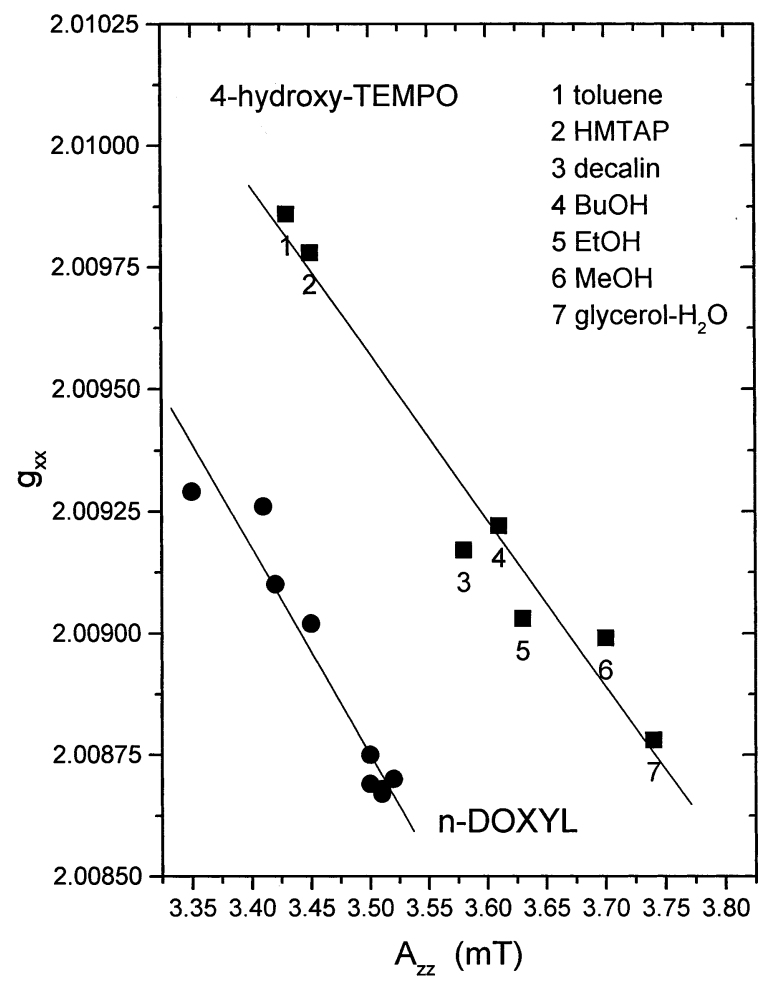

Fig. 7. Dependence of the $g_{x x}$ tensor element on the $A_{z z}$ ${ }^{14} \mathrm{~N}$-hyperfine tensor element for 4-hydroxy-2,2,6,6-tetramethylpiperidine-1-oxyl (TEMPOL) in rigid glasses of different polarities (ם). (1) toluene; (2) hexamethyltriamide phosphate; (3) decalin: (4) butanol; (5) ethanol; (6) methanol; (7) glycerol-water (1:1) mixture. Data from Ondar et al. (1985), measured at $140 \mathrm{GHz}$. Data for 1-acyl-2-[n-(4,4dimethyloxazolidine- $N$-oxyl)stearoyl] phosphatidylcholines with differing $n$ in frozen hydrated phospholipid bilayers ( $)$, measured at $250 \mathrm{GHz}$, are from Earle et al. (1994).
Because the polarity dependence of the $g$-tensor is dominated by the oxygen lone pair orbitals, it is expected that the $g_{x x}$-element will be particularly susceptible to hydrogen bonding. Data obtained for the isotropic hyperfine and $g$-values from 9 $\mathrm{GHz}$ ESR measurements already have revealed that changes in $g_{\mathrm{o}}$ relative to those in $a_{\mathrm{o}}^{\mathrm{N}}$ are greater by a factor of approximately 1.5 in hydrogen-bonding solvents than in aprotic solvents (Kawamura et al., 1967). This result was not corroborated by Griffith et al. (1974), however. Measurements of $g$-values at high fields therefore have better potential for distinguishing water-accessible (i.e., hydrogen-bonding) environments from other polar environments. An illustration is given in the following section.

\section{Polarity profile in bacteriorhodopsin}

The polarity profile of the proton channel in bacteriorhodopsin has been probed by Steinhoff et al. (2000) using high-field ESR of site-directed spin-labelled residues. The $g_{x x}$ tensor elements determined from frozen purple membrane dispersions are given as a function of the transmembrane position of the spin-labelled residue in the left-hand panel of Fig. 8. Highest polarity, i.e., minimum value of $g_{x x}$, is found for spin-labelled residue 162 in the loop between transmembrane helices $\mathrm{E}$ and F on the cytoplasmic side of the membrane, and for residue 129 in the loop between transmembrane helices $\mathrm{D}$ and $\mathrm{E}$ on the extracellular side of the membrane. These spin-labelled residues are fully exposed to the aqueous phase, because a value of $g_{x x}=2.00834$ is obtained for the spin label free in aqueous-glycerol solution. The largest value of $g_{x x}$, i.e., the minimum in polarity, is reached at residue position 46 , close to the middle of the membrane between the proton donor D96 and the retinal chromophore. In this region of the proton channel, the polarity reaches an intermediate value still considerably higher than that corresponding to hydrocarbon solvents (cf. Fig. 7).

The dependence of the $g_{x x}$-tensor element on the $A_{z z}$ element of the ${ }^{14} \mathrm{~N}$-hyperfine tensor is given in the right-hand panel of Fig. 8. Two separate linear dependences are obtained depending on which 
A
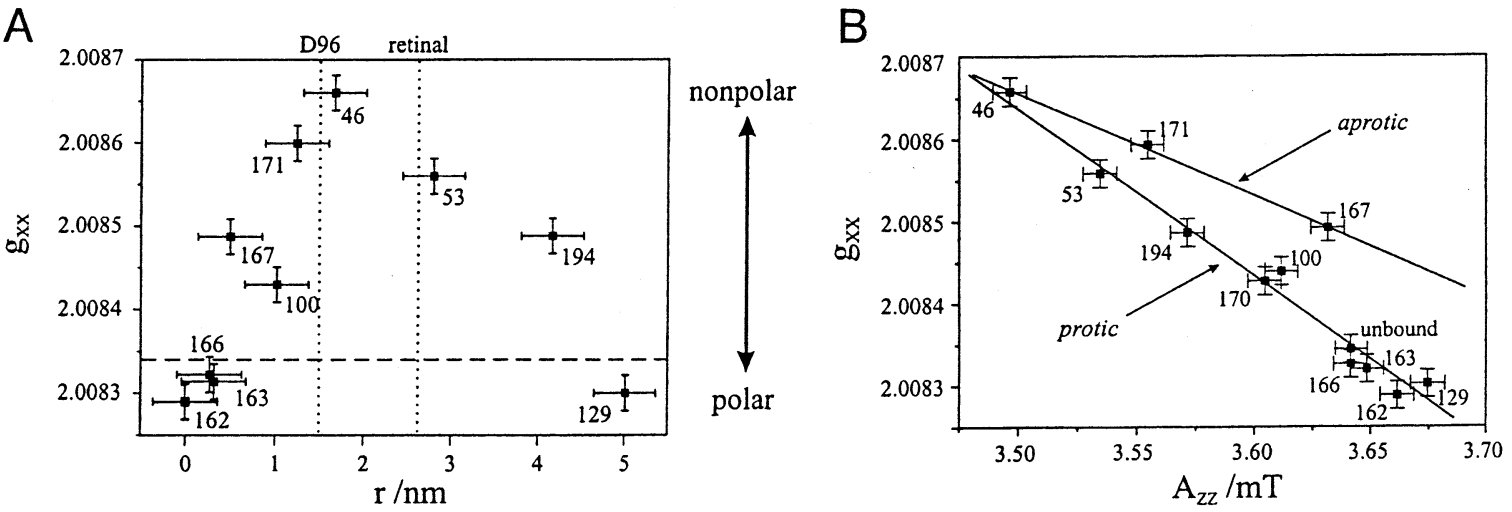

Fig. 8. Tensor elements $g_{x x}$ determined from low-temperature 95-GHz ESR spectra of bacteriorhodopsin cysteine-replacement mutants spin-labelled with PROXYL-methanethiosulphonate. The sequence position of the labelled residue is indicated on the figure. Left-hand: $g_{x x}$ as a function of the transmembrane residue position, $r$, relative to residue 164. Right-hand: $g_{x x}$ as a function of the hyperfine tensor element $A_{z z}$ of the same label (Steinhoff et al., 2000).

bacteriorhodopsin residues are spin-labelled. That for spin-labelled residues 46,171 and 167 is 1.5 times less steep than that for the other spin-labelled residues. It is assigned to residues in a more aprotic environment, on the inward-facing surfaces of helices B and F. Analysis of the crystal structure of bacteriorhodopsin is consistent with such an interpretation. Residues 46, 171 and 167 have fewer water molecules and polar residues within a radius of $1 \mathrm{~nm}$ than do the other spin-labelled residues, which are located mostly in the extramembranous loops. A converse relation is found for the number of apolar residues within a sphere of the same radius (Steinhoff et al., 2000).

\section{Conclusions}

Conventional ESR spectroscopy of spin-labelled lipids and proteins has proved extremely successful in the study of membrane structure and dynamics. High-field ESR spectroscopy now provides the potential to extend this versatility of the spin-label method. The directions in which this is possible are reviewed in the present paper. Of particular importance are the sensitivity to nonaxial rotations and lateral ordering, the polarity sensitivity of the $g_{x x}$ element of the $g$-tensor, and the enhanced sensitivity to rate and anisotropy of rapid segmental rotation. Measurements with non-frozen aqueous samples, although technically more difficult, are possible and a wide range of fruitful applications to membrane biophysics and structural membrane biology is to be anticipated in the future.

\section{Acknowledgements}

The research on high-field spin-label ESR spectroscopy from the author's laboratory is supported by the Schwerpunkt Program: High-field EPR of the Deutsche Forschungsgemeinschaft.

\section{References}

Barnes, J.P., Freed, J.H., 1997. Aqueous sample holders for high-frequency electron spin resonance. Rev. Sci. Instrum. 68, 2838-2846.

Barnes, J.P., Freed, J.H., 1998a. Dynamics and ordering in mixed model membranes of dimyristoylphosphatidylcholine and dimyristoylphosphatidylserine: a $250-\mathrm{GHz}$ electron spin resonance study using cholestane. Biophys. J. 75, 2532-2546.

Barnes, J.P., Freed, J.H., 1998b. A 'shunt' Fabry-Perot resonator for high-frequency electron spin resonance utilizing a variable coupling scheme. Rev. Sci. Instrum. 69, 30223027.

Bennati, M., Gerfen, G.J., Martinez, G.V., Griffin, R.G., Singel, D.J., Millhauser, G.L., 1999. Nitroxide side-chain dynamics in a spin-labeled helix-forming peptide revealed by high-frequency (139.5 GHz) EPR spectroscopy. J. Magn. Reson. 139, 281-286. 
Berliner, L.J. (Ed.), 1998. Spin Labeling, The Next Millenium, Biological Magnetic Resonance, vol. 14. Plenum Press, New York.

Berliner, L.J., Reuben, J. (Eds.), 1989. Biological Magnetic Resonance, vol. 8. Plenum Publishing Corp, New York.

Brown, D.A., Rose, J.K., 1992. Sorting of GPI-anchored proteins to glycolipid-enriched membrane subdomains during transport to the apical cell surface. Cell 68, 533-544.

Budil, D.E., Earle, K.A., Freed, J.H., 1993. Full determination of the rotational diffusion tensor by electron paramagnetic resonance at $250 \mathrm{GHz}$. J. Phys. Chem. 97, 1294-1303.

Cohen, A.H., Hoffman, B.M., 1973. Hyperfine interactions in perturbed nitroxides. J. Am. Chem. Soc. 95, 2061-2062.

Diamond, J.M., Katz, Y., 1974. Interpretation of nonelectrolyte partition coefficients between dimyristoyl lecithin and water. J. Membr. Biol. 17, 121-154.

Earle, K.A., Budil, D.E., Freed, J.H., 1993. 250-GHz EPR of nitroxides in the slow-motional regime-models of rotational diffusion. J. Phys. Chem. 97, 13289-13297.

Earle, K.A., Moscicki, J.K., Ge, M.T., Budil, D.E., Freed, J.H., 1994. 250-GHz electron spin resonance studies of polarity gradients along the aliphatic chains in phospholipid membranes. Biophys. J. 66, 1213-1221.

Freed, J.H., 1976. Theory of slowly tumbling ESR spectra for nitroxides. In: Berliner, L.J. (Ed.), Spin Labeling, Theory and Applications. Academic Press Inc, New York, pp. $53-132$.

Gaffney, B.J., Marsh, D., 1998. High-frequency, spin-label EPR of nonaxial lipid ordering and motion in cholesterolcontaining membranes. Proc. Natl. Acad. Sci. USA 95, $12940-12943$.

Gagua, A.V., Malenkov, G.G., Timofeev, V.P., 1978. Hydrogen-bond contribution to isotropic hyperfine splitting constant of a nitroxide free-radical. Chem. Phys. Lett. 56, $470-473$.

Goldman, A., Bruno, G.V., Polnaszek, C.F., Freed, J.H., 1972. An ESR study of anisotropic rotational reorientation and slow tumbling in liquid and frozen media. J. Chem. Phys. 56, 716-735.

Griffith, O.H., Dehlinger, P.J., Van, S.P., 1974. Shape of the hydrophobic barrier of phospholipid bilayers. Evidence for water penetration in biological membranes. J. Membr. Biol. 15, 159-192.

Gutowsky, H.S., Holm, C.H., 1956. Rate processes and nuclear magnetic resonance spectra. 2. Hindered internal rotation of amides. J. Chem. Phys. 25, 1228-1234.

Israelachvili, J., Sjösten, J., Eriksson, L.E.G., Ehrström, M., Gräslund, A., Ehrenberg, A., 1975. ESR spectral analysis of the molecular motion of spin labels in lipid bilayers and membranes based on a model in terms of two angular motional parameters and rotational correlation times. Biochim. Biophys. Acta 382, 125-141.

Karplus, M., Fraenkel, G.K., 1961. Theoretical interpretation of carbon-13 hyperfine interactions in electron spin resonance spectra. J. Chem. Phys. 25, 1312.

Kawamura, T., Matsunami, S., Yonezawa, T., 1967. Solvent effects on the $g$-value of di-t-butyl nitric oxide. Bull.
Chem. Soc. Jpn. 40, 1111-1115.

Kurad, D., Jeschke, G., Marsh, D., 2001. Spin-label HF-EPR of lipid ordering in cholesterol-containing membranes. Appl. Magn. Reson. 21, 469-481.

Lebedev, Y.S., 1992. Kinetics of radical processes by high resolution electron paramagnetic resonance. Prog. Reaction Kinetics 17, 281-328.

Lee, S., Ames, D.P., 1984. Generalized theoretical treatment of axially-symmetric electron spin resonance hyperfine centers under slow orientational diffusion motion. J. Chem. Phys. 80, 1766-1771.

Lee, S., Tang, S.Z., 1985. Comparison of different theories for slow-tumbling axial nitroxide ESR hyperfine centers in polycrystalline-amorphous substances. J. Chem. Phys. 83, 4348-4352.

Livshits, V.A., 1976. Slow anisotropic tumbling in ESR spectra of nitroxyl radicals. J. Magn. Reson. 23, 307-313.

Livshits, V.A., Marsh, D., 2000. Simulation studies of highfield EPR spectra of spin-labeled lipids in membranes. J. Magn. Reson. 147, 59-67.

Marsh, D., 1980. Molecular motion in phospholipid bilayers in the gel phase: long axis rotation. Biochemistry 19, $1632-1637$.

Marsh, D., 1981. Electron spin resonance: spin labels. In: Grell, E. (Ed.), Membrane Spectroscopy. Molecular Biology, Biochemistry and Biophysics, vol. 31. Springer, Berlin, Heidelberg, pp. 51-142.

Marsh, D., 1989. Experimental methods in spin-label spectral analysis. In: Berliner, L.J., Reuben, J. (Eds.), Biological Magnetic Resonance, vol. 8. Plenum Publishing Corp, New York, pp. 255-303.

Marsh, D., 2001. Polarity and permeation profiles in lipid membranes. Proc. Natl. Acad. Sci. USA, 98, 7777-7782.

Marsh, D., Horváth, L.I., 1989. Spin-label studies of the structure and dynamics of lipids and proteins in membranes. In: Hoff, A.J. (Ed.), Advanced EPR. Applications in Biology and Biochemistry. Elsevier, Amsterdam, pp. $707-752$.

Marsh, D., Horváth, L.I., 1998. Structure, dynamics and composition of the lipid-protein interface. Perspectives from spin-labelling. Biochim. Biophys. Acta 1376, 267296.

Marsh, D., Páli, T., Horváth, L.I., 1998. Progressive saturation and saturation transfer EPR for measuring exchange processes and proximity relations in membranes. In: Berliner, L.J. (Ed.), Spin Labeling. The Next Millenium. Plenum Press, New York, pp. 23-82.

Ondar, M.A., Grinberg, O.Y., Dubinskii, A.A., Lebedev, Y.S., 1985. Study of the effect of the medium on the magneticresonance parameters of nitroxyl radicals by high-resolution EPR spectroscopy. Sov. J. Chem. Phys. 3, 781-792.

Seelig, J., 1976. Anisotropic motion in liquid crystalline structures. In: Berliner, L.J. (Ed.), Spin Labeling. Theory and Applications. Academic Press, New York, pp. 373-409.

Seelig, J., Limacher, H., Bader, P., 1972. Molecular architecture of liquid crystalline bilayers. J. Am. Chem. Soc. 94, 6364-6371. 
Simons, K., Ikonen, E., 1997. Functional rafts in cell membranes. Nature 387, 569-572.

Smirnov, A.I., Belford, R.L., Clarkson, R.B., 1998. Comparative spin label spectra at X-band and W-band. In: Berliner, L.J. (Ed.), Spin Labeling. The Next Millenium. Plenum Press, New York, pp. 83-107.

Smirnova, T.I., Smirnov, A.I., Clarkson, R.B., Belford, R.L., 1995. W-Band (95 GHz) EPR spectroscopy of nitroxide radicals with complex proton hyperfine structure: fast motion. J. Phys. Chem. 99, 9008-9016.

Steinhoff, H.J., Savitsky, A., Wegener, C., Pfeiffer, M., Plato, M., Möbius, K., 2000. High-field EPR studies of the structure and conformational changes of site-directed spin labeled bacteriorhodopsin. Biochim. Biophys. Acta 1457, 253-262.
Stone, A.J., 1963. $g$-factors of aromatic free radicals. Mol. Phys. 6, 509-515.

Subczynski, W.K., Hyde, J.S., Kusumi, A., 1989. Oxygen permeability of phosphatidylcholine-cholesterol membranes. Proc. Natl. Acad. Sci. USA 86, 4474-4478.

Subczynski, W.K., Wisniewska, A., Yin, J.J., Hyde, J.S., Kusumi, A., 1994. Hydrophobic barriers of lipid bilayer membranes formed by reduction of water penetration by alkyl chain unsaturation and cholesterol. Biochemistry 33, 7670-7681.

Wiener, M.C., White, S.H., 1992. Structure of a fluid dioleoylphosphatidylcholine bilayer determined by joint refinement of X-ray and neutron diffraction data. II. Distribution and packing of terminal methyl groups. Biophys. J. $61,428-433$. 